\title{
COURSE 5
}

\section{QUANTUM TRANSPORT IN SEMICONDUCTOR-SUPERCONDUCTOR MICROJUNCTIONS}

\author{
C.W.J. Beenakker \\ Instituut-Lorentz, Untverstty of Leiden \\ 2300 RA Leiden, The Netherlands
}

E Akkermans, G Montambaux, J-L Plchard and J Zinn-Justın, eds Les Houches, Session LXI, 1994

Physıque Quantıque Mésoscopıque

Mesoscoptc Quantum Physics

(C) 1995 Elsevier Science B V All rights reserved 


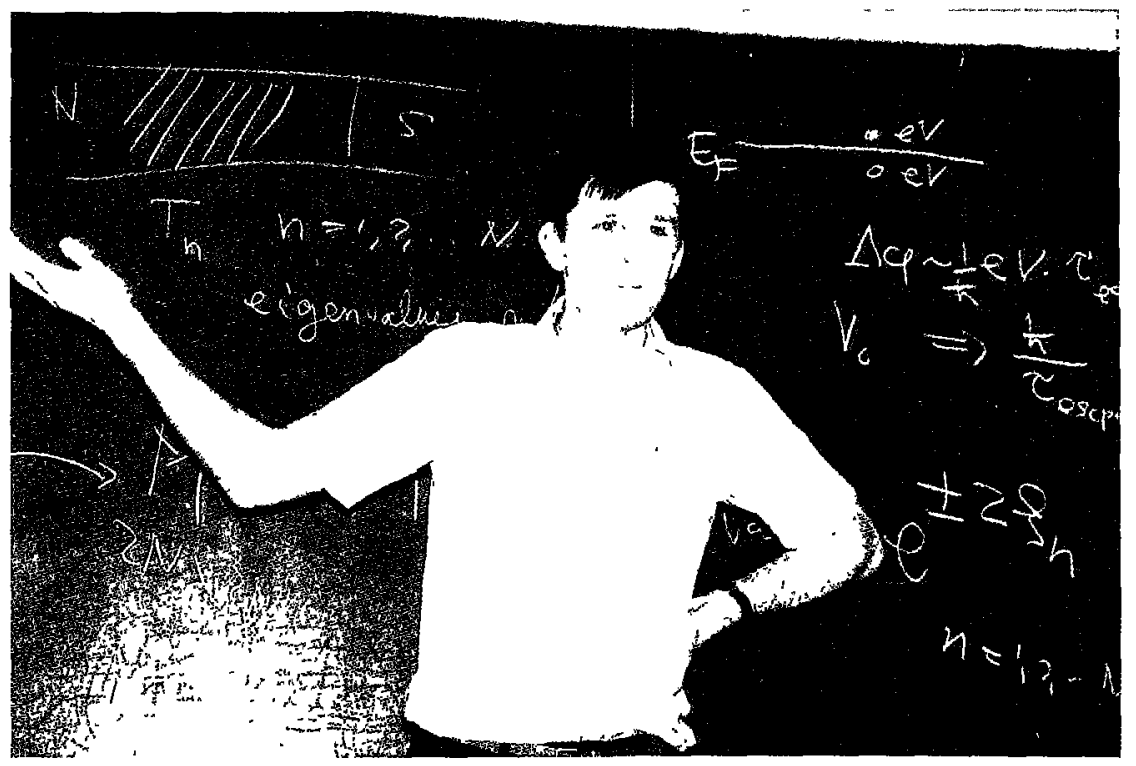




\section{Contents}

1 Introduction 283

2 Scattering theory 286

3 Three simple applications $\quad 292$

31 Quantum point contact 292

32 Quantum dot 293

33 Disordered junction $\quad 295$

$\begin{array}{ll}4 \text { Weak localization } & 297\end{array}$

5 Reflectionless tunneling $\quad 300$

51 Numerical simulations $\quad 300$

52 Scaling theory $\quad 303$

53 Double-batrier junction 308

54 Circult theory 311

6 Unıversal conductance fluctuations $\quad 316$

$\begin{array}{llr}7 & \text { Shot norse } & 318\end{array}$

8 Conclusion $\quad 320$

$\begin{array}{lr}\text { References } & 322\end{array}$ 
$\therefore$

1 


\section{Introduction}

At the interface between a normal metal and a superconductor, dissipative electical current is converted into dissipationless supercurrent The mechanism for this conversion was discovered thirty years ago by A F Andreev [1] An electron excitation slightly above the Fermi level in the normal metal is reflected at the interface as a hole excitation slightly below the Fermı level (see fig 1) The missing charge of $2 e$ is removed as a supercurrent The reflected hole has (approximately) the same momentum as the incident electron (The two momenta ale precisely equal at the Fermı level) The velocity of the hole is minus the velocity of the electron (cf the notion of a hole as a "time-1eversed" electron) This currous scattering process is known as retro-reflection or Andreev reflection

The early theoretical work on the conductance of a normal-metal - superconductor (NS) junction treats the dynamics of the quasiparticle excitations semclassically, as is appropriate for macroscopic junctions Phase coherence of the electrons and the Andreev-reflected holes is ignored Interest in "mesoscopic" NS junctions, where phase coherence plays an important role, is a recent development Significant advances have been made during the last few years in our understanding of quantum interference effects due to phase-coherent Andreev reflection Much of the motivation has come from the technological advances in the fabrication of a highly transparent contact between a superconducting film and the two-dimensional electron gas in a semiconductor heterostructure These systems are ideal for the study of the interplay of Andreev reflection and the mesoscopic effects known to occur in semiconductor nanostructures [2], because of the large Fermi wavelength, large mean free path, and because of the possibility to confine the carriers electrostatically by means of gate electrodes In this series of lectures we review the present status of this rapidly developing field of research

To apprecidte the importance of phase coherence in NS junctions, consider the resistance of a normal-metal wire (length $L$, mean free path $l$ ) This resistance increases monotonically with $L$ Now attach the wire to a superconductor via a tunnel barrer (transmission probability $\Gamma$ ) Then the resistance has a minımum when $L \simeq l / \Gamma$ The minimum disappears if the phase coherence between the electrons and holes is destroyed, by increasing the voltage or by applying a magnetic field The resistance minımum is associated with the crossover from a $\Gamma^{-1}$ 

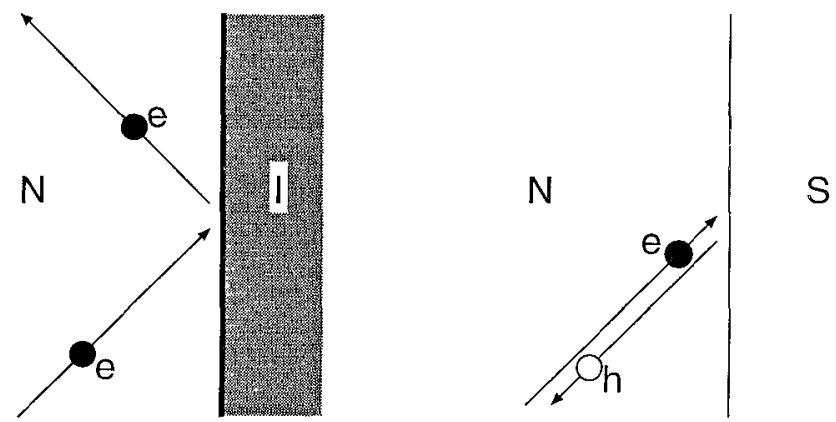

Fig I Noimal ieflection by an insulator (I) veisus Andreev teflection by a superconductor (S) of an election excitation in a normal metal (N) near the Ferm level Normal ieflection (left) conserves charge but does not conserve momentum Andieev reflection (1ight) conserves momentum but does not conserve chatge The election (c) is reflected as a hole (h) with the same momentum and opposite velocity The missing charge of $2 e$ is absoibed as a Cooper par by the superconducting condensate

to a $\Gamma^{-2}$ dependence on the barrier transparency The $\Gamma^{-2}$ dependence is as expected for tunneling into a superconductor, being a two-particle process The $\Gamma^{-1}$ dependence is surprising It is as if the Andreev-reflected hole can tunnel through the barıer without reflections This so called "reflectionless tunneling" requies telatively transparent NS interfaces, with $\Gamma \gtrsim l / L$ Semiconductor - superconductor junctions are convenient, since the Schottky barrier at the interface is much more transparent than a typical dielectric tunnel barrier The technological effort is directed towards making the interface as tiansparent as possible A nearly ideal NS inter face $(\Gamma \simeq 1)$ is 1equired if one wishes to study how Andieev reflection modifies the quantum interference effects in the normal state (Fo1 $I \ll 1$ these are obscured by the much larger reflectionless-tunneling effect ) The modificatıons can be quite remarkable We discuss two examples

The first is weak localization In the normal state, weak localization can not be detected in the current-voltage $(I-V)$ charactenstic, but requires application of a magnetic field The reason is that application of a voltage (in contrast to a magnetic field) does not break time-reversal symmetry In an NS junction, however, weak localization can be detected in the $I-V$ characteristic, because application of a voltage destroys the phase coherence between electrons and holes The result is a small dip in $\partial I / \partial V$ versus $V$ around $V=0$ for $\Gamma \simeq 1$ On reducing $\Gamma$, the dip crosses over to a peak due to reflectionless tunneling The peak is much laigei than the dip, but the widths are approximately the same

The second example is universal conductance fluctuations In the normal state, the conductance fluctuates from sample to sample with a variance which is independent of sample size or degree of disorder This is one aspect of the univeisality The other aspect is that breaking of time-reversal symmetry (by a magnetic field) 
reduces the variance by precisely a factor of two In an NS junction, the conductance fluctuations are also size and disorder independent However, application of a time-reversal-symmetry breakıng magnetic field has no effect on the magnitude

These three phenomena, weak localization, reflectionless tunneling, and unıversal conductance fluctuations, are discussed in sections 4, 5, and 6, respectively Sections 2 and 3 are devoted to a description of the theoretical method and to a tew illustrative applications The method is a scattering theory, which relates the conductance $G_{N S}$ of the NS junction to the $N \times N$ transmission matrix $t$ in the notmal state ( $N$ is the number of transverse modes at the Fermi level) In the limit of zero temperature, zero voltage, and zero magnetıc field, the relationship IS

$$
G_{N S}=\frac{4 e^{2}}{h} \sum_{n=1}^{N} \frac{T_{n}^{2}}{\left(2-T_{n}\right)^{2}}
$$

where the transmission eigenvalue $T_{n}$ is an eigenvalue of the matrix product $t t^{\dagger}$ The same numbers $T_{n}(n=1,2, \quad, N)$ determine the conductance $G_{\mathrm{N}}$ in the normal state, according to the Landauer formula

$$
G_{\mathrm{N}}=\frac{2 e^{2}}{h} \sum_{n=1}^{N} T_{n}
$$

The fact that the same transmission eigenvalues determine both $G_{\mathrm{N}}$ and $G_{\mathrm{NS}}$ means that one can use the same (numerical and analytical) techniques developed for quantum transport in the normal state This is a substantial technical and conceptual simplification

The scattering theory can also be used for other transport properties, other than the conductance, both in the normal and the superconducting state An example, discussed in section 7, is the shot noise due to the discreteness of the carriers $A$ doubling of the ratio of shot-noise power to current can occur in an NS junction, consistent with the notion of Cooper pair transport in the superconductor

We conclude in section 8

We restrict ourselves in this review (with one exception) to two-terminal geometiles, with a single NS interface Equation (1 1), as well as the Landauer formula (1 2), only describe the two-terminal conductance More complex multiterminal geometries, involving several NS interfaces, have been studied theoretIcally by Lambert and coworkers [3,4], and experimentally by Petrashov et al [5] Since we focus on phase-coherent effects, most of our discussion concerns the linear-response regime of infinitesimal applied voltage A recent review by Klapwijk contains a more extensive coverage of the non-linear response at higher voltages [6] The scatterıng approach has also been applied to the Josephson effect in SNS junctions [7], resulting in a formula for the supercurrent-phase relation- 
ship in terms of the transmission eigenvalues $T_{n}$ in the normal state We do not discuss the Josephson effect here, but refer to ref [8] for a review of mesoscopic SNS junctions Taken together, ref [8] and the present work describe a unified approach to mesoscopic superconductıvity

\section{Scattering theory}

The model considered is illustrated in fig 2 It consists of a disordered normal region (hatched) adjacent to a superconductor (S) The disordered region may also contain a geometrical constriction or a tunnel barrier To obtan a well-defined scatterıng problem we insert ideal (impurity-free) normal leads $\mathrm{N}_{1}$ and $\mathrm{N}_{2}$ to the left and right of the disordered region The NS interface is located at $x=0$ We assume that the only scattering in the superconductor consists of Andreev reflection at the NS interface, $1 \mathrm{e}$ we consider the case that the disorder is contained entırely within the normal region The spatial separation of Andreev and noimal scattering is the key simplification which allows us to relate the conductance directly to the normal-state scattering matrix The model is directly applicable to a superconductor in the clean limit (mean free path in S large compared to the superconductıng coherence length $\xi$ ), or to a point-contact junction (formed by a constıction which is narrow compared to $\xi$ ) In both cases the contribution of scattering within the superconductor to the junction resistance can be neglected [9]

The scattering states at energy $\varepsilon$ are eigenfunctions of the Bogoliubov-de Gennes $(\mathrm{BdG})$ equation This equation has the form of two Schrodinger equations for electron and hole wavefunctions $\mathrm{u}(\boldsymbol{r})$ and $\mathrm{v}(r)$, coupled by the pail potential $\Delta(\boldsymbol{r})[10]$

$$
\left(\begin{array}{cc}
\mathcal{H}_{0} & \Delta \\
\Delta^{*} & -\mathcal{H}_{0}^{*}
\end{array}\right)\left(\begin{array}{l}
\mathrm{u} \\
\mathrm{v}
\end{array}\right)=\varepsilon\left(\begin{array}{l}
\mathrm{u} \\
\mathrm{v}
\end{array}\right)
$$

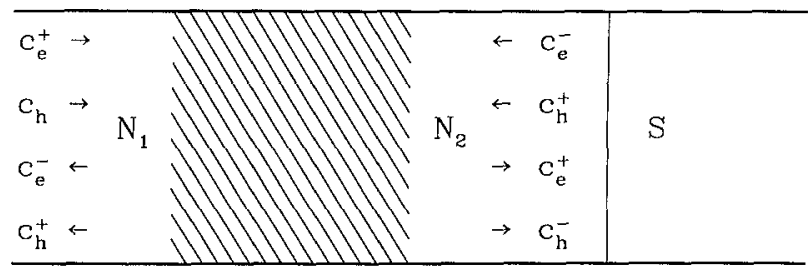

$\longrightarrow 0 \rightarrow x$

Fig 2 Noimal metal-superconductor junction contaning a disordeted normal tegion (hatched) Scatteing states in the two normal leads $N_{1}$ and $N_{2}$ are indicated schematically 
Here $\mathcal{H}_{0}=(p+e \boldsymbol{A})^{2} / 2 m+V-E_{\Gamma}$ is the sungle-electron Hamiltonian, containing an electrostatic potential $V(\boldsymbol{r})$ and vector potential $A(\boldsymbol{r})$ The excitation eneigy $\varepsilon$ is measured relative to the Fermı energy $E_{\mathrm{F}}$ To simplify construction of the scattering basis we assume that the magnetic field $B$ (in the $z$-direction) vanishes outside the disordered region One can then choose a gauge such that $\boldsymbol{A} \equiv 0$ in lead $\mathrm{N}_{2}$ and in $\mathrm{S}$, while $A_{x}, A_{z}=0, A_{y}=A_{1} \equiv$ constant in lead $\mathrm{N}_{1}$

The parr potential in the bulk of the superconductor $(x \gg \xi)$ has amplitude $\Delta_{0}$ and phase $\phi$ The spatial dependence of $\Delta(r)$ near the NS interface is determined by the self-consistency relation [10]

$$
\Delta(\boldsymbol{r})=|g(\boldsymbol{r})| \sum_{\varepsilon>0} \mathrm{v}^{*}(\boldsymbol{r}) \mathrm{u}(\boldsymbol{r})[1-2 f(\varepsilon)]
$$

where the sum is over all states with positive eigenvalue, and $f(\varepsilon)=[1+$ $\left.\exp \left(\varepsilon / k_{\mathrm{B}} T\right)\right]^{-1}$ is the Fermi function The coefficient $g$ is the interaction constant of the BCS theory of superconductivity At an NS inter face, $g$ diops abruptly (over atomic distances) to zero, in the assumed absence of any parring interaction in the normal region Therefore, $\Delta(\boldsymbol{r}) \equiv 0$ for $x<0$ At the superconducting side of the NS interface, $\Delta(r)$ recovers its bulk value $\Delta_{0} \mathrm{e}^{1 \phi}$ only at some distance from the interface We will neglect the suppression of $\Delta(r)$ on approaching the NS interface, and use the step-function model

$$
\Delta(\boldsymbol{r})=\Delta_{\mathrm{o}} \mathrm{e}^{\mathrm{l} \phi} \theta(x)
$$

This model is also reterred to in the literature as a "rigid boundary-condition" Likhaiev [11] discusses in detail the conditions for its validity If the width $W$ of the NS junction is small compared to $\xi$, the non-uniformities in $\Delta(r)$ extend only over a distance of order $W$ fiom the junction (because of "geometical dilution" of the influence of the nariow junction in the wide superconductor) Since non-uniformities on length scales $\ll \xi$ do not affect the dynamics of the quasiparticles, these can be neglected and the step-function model holds A point contact or microbridge belongs in general to this class of junctions Alternatively, the step-function model holds also for a wide junction if the iesistivity of the junction region is much bigger than the resistivity of the bulk superconductor This condition is formulated more precisely in ref [11] A semiconductor - superconductor junction is typically in this second category Note that both the two cases are consistent with our assumption that the disorder is contained entirely within the normal region

It is worth emphasizing that the absence of a pairing interaction in the noimal region $(g(r) \equiv 0$ for $x<0)$ implies a vanıshing pair potentral $\Delta(r)$, accoiding to eq (2), but does not imply a vanıshing order parameter $\Psi(\boldsymbol{r})$, which is given 
by

$$
\Psi(\boldsymbol{r})=\sum_{\varepsilon>0} \mathrm{v}^{*}(\boldsymbol{r}) \mathrm{u}(\boldsymbol{r})[1-2 f(\varepsilon)]
$$

Phase coherence between the electron and hole wave functions $u$ and $v$ leads to $\Psi(r) \neq 0$ for $x<0$ The term "proximity effect" can therefore mean two different things One is the suppression of the pair potential $\Delta$ at the superconducting side of the NS interface This is a small effect which is neglected in the present work (and in most other papers in this field) The other is the induction of a nonzeio order parameter $\Psi$ at the normal side of the NS interface This effect is fully included here, even though $\Psi$ does not appear explicitly in the expiessions which follow The reason is that the order parameter quantifies the degree of phase coherence between electrons and holes, but does not itself affect the dynamics of the quasiparticles (The BdG equation (2 1) contains $\Delta$ not $\Psi$ )

We now construct a basis for the scatteing matrix ( $s$ matrix) In the noimal lead $\mathrm{N}_{2}$ the eigenfunctions of the BdG equation (2 1) can be written in the form

$$
\begin{aligned}
& \psi_{n \mathrm{e}}^{ \pm}\left(\mathrm{N}_{2}\right)=\left(\begin{array}{l}
1 \\
0
\end{array}\right)\left(k_{n}^{\mathrm{e}}\right)^{-1 / 2} \Phi_{n}(y, z) \exp \left( \pm_{1} k_{n}^{\mathrm{e}} x\right) \\
& \psi_{n \mathrm{~h}}^{ \pm}\left(\mathrm{N}_{2}\right)=\left(\begin{array}{c}
0 \\
1
\end{array}\right)\left(k_{n}^{\mathrm{h}}\right)^{-1 / 2} \Phi_{n}(y, z) \exp \left( \pm_{\mathbf{l}} k_{n}^{\mathrm{h}} x\right),
\end{aligned}
$$

where the wavenumbers $k_{n}^{\mathrm{e}}$ and $k_{n}^{\mathrm{h}}$ are given by

$$
k_{n}^{\mathrm{e} h} \equiv\left(2 m / \hbar^{2}\right)^{1 / 2}\left(E_{\mathrm{F}}-E_{n}+\sigma^{\mathrm{eh}} \varepsilon\right)^{1 / 2},
$$

and we have defined $\sigma^{\mathrm{e}} \equiv 1, \sigma^{\mathrm{h}} \equiv-1$ The labels e and $\mathrm{h}$ indicate the election or hole character of the wavefunction The index $n$ labels the modes, $\Phi_{n}(y, z)$ is the transverse wavefunction of the $n$-th mode, and $E_{n}$ its threshold energy

$$
\left[\left(p_{y}^{2}+p_{z}^{2}\right) / 2 m+V(y, z)\right] \Phi_{n}(y, z)=E_{n} \Phi_{n}(y, z)
$$

The eigenfunction $\Phi_{n}$ is normalized to unity, $\int \mathrm{d} y \int \mathrm{d} z\left|\Phi_{n}\right|^{2}=1$ With this normalization each wavefunction in the basis (2 5) carries the same amount of quasiparticle current The eigenfunctions in lead $N_{1}$ are chosen similarly, but with an additional phase factor $\exp \left[-1 \sigma^{\mathrm{e} h}\left(e A_{1} / \hbar\right) y\right]$ from the vector potential

A wave incident on the disordered normal region is described in the basis (2 5) by a vector of coefficients

$$
c_{\mathrm{N}}^{1 \mathrm{n}} \equiv\left(c_{\mathrm{e}}^{+}\left(\mathrm{N}_{1}\right), c_{\mathrm{e}}^{-}\left(\mathrm{N}_{2}\right), c_{\mathrm{h}}^{-}\left(\mathrm{N}_{1}\right), c_{\mathrm{h}}^{+}\left(\mathrm{N}_{2}\right)\right)
$$

(The mode-index $n$ has been suppressed for simplicity of notation ) The ieflected and tiansmitted wave has vector of coefficients

$$
c_{\mathrm{N}}^{\text {out }} \equiv\left(c_{\mathrm{e}}^{-}\left(\mathrm{N}_{1}\right), c_{\mathrm{e}}^{+}\left(\mathrm{N}_{2}\right), c_{\mathrm{h}}^{+}\left(\mathrm{N}_{1}\right), c_{\mathrm{h}}^{-}\left(\mathrm{N}_{2}\right)\right)
$$


The $s$-matrix $s_{\mathrm{N}}$ of the normal region relates these two vectors,

$$
c_{\mathrm{N}}^{\text {out }}=s_{\mathrm{N}} c_{\mathrm{N}}^{\text {in }}
$$

Because the normal region does not couple electrons and holes, this matrix has the block-diagonal form

$$
s_{N}(\varepsilon)=\left(\begin{array}{cc}
s_{0}(\varepsilon) & 0 \\
0 & s_{0}(-\varepsilon)^{*}
\end{array}\right), s_{0} \equiv\left(\begin{array}{cc}
r_{11} & t_{12} \\
t_{21} & r_{22}
\end{array}\right)
$$

Heie $s_{0}$ is the unitary $s$-matrix associated with the single-electron Hamiltonian $\mathcal{H}_{0}$ The ieflection and transmission matrices $r(\varepsilon)$ and $t(\varepsilon)$ are $N \times N$ matıces, $N(\varepsilon)$ being the number of propagatıng modes at energy $\varepsilon$ (We assume for simplicity that the number of modes in leads $\mathrm{N}_{1}$ and $\mathrm{N}_{2}$ is the same ) The matrix $s_{0}$ is unitary $\left(s_{0}^{\dagger} s_{0}=1\right)$ and satısfies the symmetry ielation $s_{0}(\varepsilon, B)_{\imath \jmath}=s_{0}(\varepsilon,-B)_{\jmath \jmath}$

For energies $0<\varepsilon<\Delta_{0}$ there are no propagating modes in the superconductor We can then define an $s$-matrix for Andreev reflection at the NS interface which 1elates the vector of coefficients $\left(c_{\mathrm{e}}^{-}\left(\mathrm{N}_{2}\right), c_{\mathrm{h}}^{+}\left(\mathrm{N}_{2}\right)\right)$ to $\left(c_{\mathrm{e}}^{+}\left(\mathrm{N}_{2}\right), c_{\mathrm{h}}^{-}\left(\mathrm{N}_{2}\right)\right)$ The elements of this $s$-matrix can be obtained by matching the wavefunctions (25) at $x=0$ to the decaying solutions in $\mathrm{S}$ of the $\mathrm{BdG}$ equation If terms of order $\Delta_{\circ} / E_{\Gamma}$ are neglected (the so called Andreev approximation [1]), the result is simply

$$
\begin{aligned}
& c_{\mathrm{e}}^{-}\left(\mathrm{N}_{2}\right)=\alpha \mathrm{e}^{\mathrm{l} \phi} c_{\mathrm{h}}^{-}\left(\mathrm{N}_{2}\right), \\
& c_{\mathrm{h}}^{+}\left(\mathrm{N}_{2}\right)=\alpha \mathrm{e}^{-1 \phi} c_{\mathrm{e}}^{+}\left(\mathrm{N}_{2}\right),
\end{aligned}
$$

where $\alpha \equiv \exp \left[-1 \arccos \left(\varepsilon / \Delta_{\mathrm{o}}\right)\right]$ Andreev reflection tianstorms an election mode into a hole mode, without change of mode index The transformation is accompanied by a phase shift, which consists of two paits

(1) A phase shift $-\arccos \left(\varepsilon / \Delta_{0}\right)$ due to the penetration of the wavefunction into the superconductor

(11) A phase shift equal to plus or minus the phase of the parr potentral in the superconductor (plus tor ieflection fiom hole to election, minus for the reverse process)

We can combine the $2 N$ linear telations (2 12) with the $4 N$ relations (2 10) to obtain a set of $2 N$ linear relations between the incident wave in lead $\mathrm{N}_{1}$ and the reflected wave in the same lead

$$
\begin{aligned}
& c_{\mathrm{e}}^{-}\left(\mathrm{N}_{1}\right)=s_{\mathrm{ec}} c_{\mathrm{e}}^{+}\left(\mathrm{N}_{1}\right)+s_{\mathrm{eh}} c_{\mathrm{h}}^{-}\left(\mathrm{N}_{1}\right), \\
& c_{\mathrm{h}}^{+}\left(\mathrm{N}_{1}\right)=s_{\mathrm{he}} c_{\mathrm{e}}^{+}\left(\mathrm{N}_{1}\right)+s_{\mathrm{hh}} c_{\mathrm{h}}^{-}\left(\mathrm{N}_{1}\right)
\end{aligned}
$$

The four $N \times N$ matıces $s_{\mathrm{ee}}, s_{\mathrm{hh}}, s_{\mathrm{eh}}$, and $s_{\mathrm{he}}$ form together the scatten matrix $s$ of the whole system for energies $0<\varepsilon<\Delta_{0}$ An electron incident in lead $N_{\perp}$ is reflected either as an election (with scattering amplitudes $s_{\mathrm{ee}}$ ) or as a 
hole (with scattering amplitudes $s_{\text {he }}$ ) Similarly, the matrices $s_{\text {hh }}$ and $s_{\text {eh }}$ contain the scattering amplitudes for reflection of a hole as a hole or as an electron After some algebra we find for these matrices the expressions

$$
\begin{aligned}
& s_{\mathrm{ee}}(\varepsilon)=r_{11}(\varepsilon)+\alpha^{2} t_{12}(\varepsilon) r_{22}^{*}(-\varepsilon) M_{\mathrm{e}} t_{21}(\varepsilon), \\
& s_{\mathrm{hh}}(\varepsilon)=r_{11}^{*}(-\varepsilon)+\alpha^{2} t_{12}^{*}(-\varepsilon) r_{22}(\varepsilon) M_{\mathrm{h}} t_{21}^{*}(-\varepsilon), \\
& s_{\mathrm{eh}}(\varepsilon)=\alpha \mathrm{e}^{1 \phi} t_{12}(\varepsilon) M_{\mathrm{h}} t_{21}^{*}(-\varepsilon), \\
& s_{\mathrm{he}}(\varepsilon)=\alpha \mathrm{e}^{-1 \phi} t_{12}^{*}(-\varepsilon) M_{\mathrm{e}} t_{21}(\varepsilon),
\end{aligned}
$$

where we have defined the matrices

$$
\begin{aligned}
& M_{\mathrm{e}} \equiv\left[1-\alpha^{2} r_{22}(\varepsilon) r_{22}^{*}(-\varepsilon)\right]^{-1}, \\
& M_{\mathrm{h}} \equiv\left[1-\alpha^{2} r_{22}^{*}(-\varepsilon) r_{22}(\varepsilon)\right]^{-1}
\end{aligned}
$$

One can verify that the $s$-matrix constructed from these four sub-matrices satisfies unitarity $\left(s^{\dagger} s=1\right)$ and the symmetry relation $s(\varepsilon, B, \phi)_{\imath \jmath}=s(\varepsilon,-B,-\phi)_{\jmath \imath}$, as required by quasiparticle-current conservation and by time-reversal invariance, respectively

For the linear-response conductance $G_{\mathrm{NS}}$ of the NS junction at zero temperature we only need the $s$-matrix at the Fermı level, i e at $\varepsilon=0$ We restrict ourselves to this case and omit the argument $\varepsilon$ in what follows We apply the general formula [12-14]

$$
G_{\mathrm{NS}}=\frac{2 e^{2}}{h} \operatorname{Tr}\left(1-s_{\mathrm{ee}} s_{\mathrm{ee}}^{\dagger}+s_{\mathrm{he}} s_{\mathrm{he}}^{\dagger}\right)=\frac{4 e^{2}}{h} \operatorname{Tr} s_{\mathrm{he}} s_{\mathrm{he}}^{\dagger}
$$

The second equality follows from unitarity of $s$, which implies $1-s_{\mathrm{ee}} s_{\mathrm{ee}}^{\dagger}=$ $s_{\mathrm{eh}} s_{\mathrm{eh}}^{\dagger}=\left(s_{\mathrm{ce}}^{\dagger}\right)^{-1} s_{\mathrm{he}}^{\dagger} s_{\mathrm{he}} s_{\mathrm{ee}}^{\dagger}$, so that $\operatorname{Tr}\left(1-s_{\mathrm{ee}} s_{\mathrm{ee}}^{\dagger}\right)=\operatorname{Tr} s_{\mathrm{he}} s_{\mathrm{he}}^{\dagger}$ We now subst1tute eq (2 17) for $\varepsilon=0(\alpha=-1)$ into eq (2 19), and obtain the expression

$$
G_{\mathrm{NS}}=\frac{4 e^{2}}{h} \operatorname{Tr} t_{12}^{\dagger} t_{12}\left(1+r_{22}^{*} r_{22}\right)^{-1} t_{21}^{*} t_{21}^{\mathrm{T}}\left(1+r_{22}^{\dagger} r_{22}^{\mathrm{T}}\right)^{-1},
$$

where $M^{\mathrm{T}} \equiv\left(M^{*}\right)^{\dagger}$ denotes the transpose of a matrix The advantage of eq (2 20) over eq (2 19) is that the former can be evaluated by using standard techniques developed for quantum transport in the normal state, since the only input is the normal-state scattering matrix The effects of multiple Andieev reflections are fully incorporated by the two matrix inversions in eq (2 20)

In the absence of a magnetic field the general formula (2 20) simplifies considerably Since the $s$-matrix $s_{0}$ of the normal region is symmetric for $B=0$, one has $r_{22}=r_{22}^{\mathrm{T}}$ and $t_{12}=t_{21}^{\mathrm{T}}$ Equation (2 20) then takes the form

$$
G_{\mathrm{NS}}=\frac{4 e^{2}}{h} \operatorname{Tr} t_{12}^{\dagger} t_{12}\left(1+r_{22}^{\dagger} r_{22}\right)^{-1} t_{12}^{\dagger} t_{12}\left(1+r_{22}^{\dagger} r_{22}\right)^{-1}
$$




$$
=\frac{4 e^{2}}{h} \operatorname{Tr}\left(t_{12}^{\dagger} t_{12}\left(2-t_{12}^{\dagger} t_{12}\right)^{-1}\right)^{2}
$$

In the second equality we have used the unitarity relation $r_{22}^{\dagger} r_{22}+t_{12}^{\dagger} t_{12}=$ 1 The trace (2 21) depends only on the eigenvalues of the Hermitian matix $t_{12}^{\dagger} t_{12}$ We denote these eigenvalues by $T_{n}(n=1,2, \quad N)$ Since the matrices $t_{12}^{\dagger} t_{12}, t_{12} t_{12}^{\dagger}, t_{21}^{\dagger} t_{21}$, and $t_{21} t_{21}^{\dagger}$ all have the same set of eigenvalues, we can omit the indices and write simply $t t^{\dagger}$ We obtain the following relation between the conductance and the transmission eigenvalues

$$
G_{\mathrm{NS}}=\frac{4 e^{2}}{h} \sum_{n=1}^{N} \frac{T_{n}^{2}}{\left(2-T_{n}\right)^{2}}
$$

This is the central iesult of ref [15]

Equation (2 22) holds for an arbitrary transmission matrix $t, 1$ e for arbitrary disorder potential It is the mult -channel generalization of a formula first obtained by Blonder, Tinkham, and Klapwijk [12] (and subsequently by Shelankov [16] and by Zaitsev [17]) for the single channel case (appropuate for a geometry such as a planar tunnel barrier, where the different scattering channels are uncoupled) A formula of similar generality for the normal-metal conductance $G_{\mathrm{N}}$ is the multıchannel Landauer formula

$$
G_{\mathrm{N}}=\frac{2 e^{2}}{h} \operatorname{Tr} t t^{\dagger} \equiv \frac{2 e^{2}}{h} \sum_{n=1}^{N} T_{n}
$$

In contrast to the Landauer formula, eq (222) for the conductance of an NS junction is a non linear function of the transmission eigenvalues $T_{n}$ When dealing with a non-linear multi channel formula as eq (2 22), it is of importance to distınguish between the transmission eigenvalue $T_{n}$ and the modal transmission probability $\mathcal{T}_{n} \equiv \sum_{n-1}^{N}\left|t_{n m}\right|^{2}$ The former is an eigenvalue of the matıx $t t^{\dagger}$, the latter a diagonal element of that matrix The Landauer formula (2 23) can be written equivalently as a sum over eigenvalues or as sum over modal transmission probabilities

$$
\frac{h}{2 e^{2}} G_{\mathrm{N}}=\sum_{n=1}^{N} T_{n} \equiv \sum_{n=1}^{N} \mathcal{T}_{n}
$$

This equivalence is of importance for (numerical) evaluations of the Landauer formula, in which one calculates the probability that an electron injected in mode $n$ is transmitted, and then obtains the conductance by summing over all modes The non-linear scattering formula (2 22), in contrast, can not be written in terms of modal transmission probabilities alone The off-diagonal elements of $t t^{\dagger}$ contribute to $G_{N S}$ in an essential way Previous attempts to generalize the one- 
dimensional Blonder-Tinkham-Klapwijk formula to more dimensions by summing over modal tiansmission probabilities (or, equivalently, by angula aveıaging) were not successful precisely because only the diagonal elements of $t t^{\dagger}$ were considered

\section{Three simple applications}

To illustrate the power and generality of the scattering formula (2 22), we discuss in this section three simple applications to the ballistic, resonant-tunneling, and diffusive transport regimes [15]

\section{Quantum point contact}

Consider first the case that the normal metal consists of a ballistic constriction with a normal-state conductance quantized at $G_{\mathrm{N}}=2 N_{0} e^{2} / h$ (a quantum point contact) The integer $N_{0}$ is the number of occupied one-dimensional subbands (per spin direction) in the constriction, or alternatively the number of transverse modes at the Fermi level which can propagate through the constriction Note that $N_{0} \ll N$ An "ideal" quantum point contact is characterized by a special set of transmission eigenvalues, which are equal to either zero or one [2]

$$
T_{n}= \begin{cases}1 & \text { if } 1 \leq n \leq N_{0}, \\ 0 & \text { if } N_{0}<n \leq N,\end{cases}
$$

where the ergenvalues have been ordered from large to small We emphasize that eq (3 1) does not imply that the transport through the constriction is adiabatic In the case of adiabatic transport, the transmission eigenvalue $T_{n}$ is equal to the modal transmission probability $\mathcal{T}_{n}$ In the absence of adiabaticity there is no direct relation between $T_{n}$ and $\mathcal{T}_{n}$ Substitution of eq (31) into eq (22) yrelds

$$
G_{\mathrm{NS}}=\frac{4 e^{2}}{h} N_{0}
$$

The conductance of the NS junction is quantized in units of $4 e^{2} / h$ This is twice the conductance quantum in the normal state, due to the current-doubling effect of Andreev reflection [18]

In the classical limit $N_{0} \rightarrow \infty$ we recover the well known result $G_{\mathrm{NS}}=2 G_{\mathrm{N}}$ for a classical ballistic point contact $[12,16,19]$ In the quantum regime, however, the simple factor-of-two enhancement only holds for the conductance plateaus, where eq (3 1) applies, and not to the transition region between two subsequent plateaus of quantized conductance To illustrate this, we compare in fig 3 the conductances $G_{\mathrm{NS}}$ and $2 G_{\mathrm{N}}$ for Buttıker's model [20] of a saddle-point constuctıon in a two-dimensional electron gas Appreciable differences appear in the 


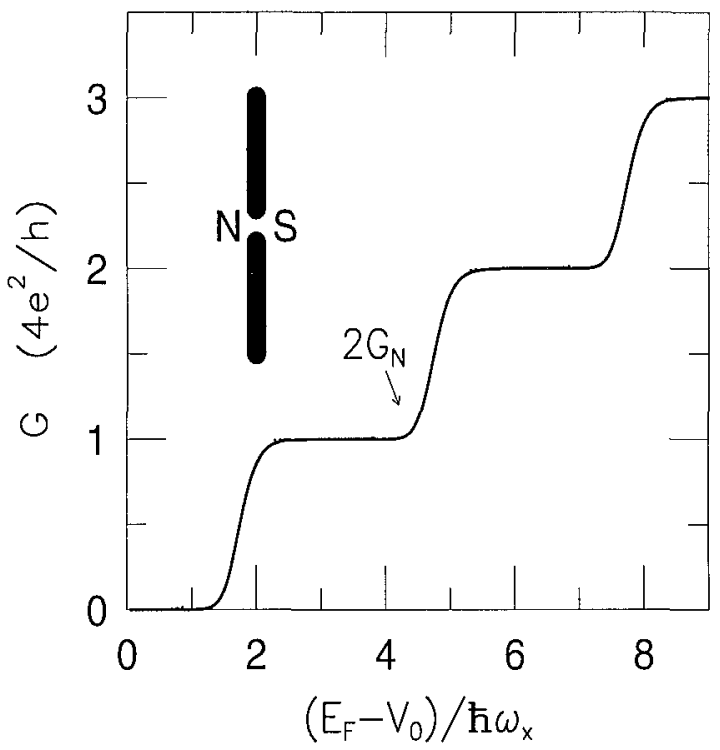

Fig 3 Solid curve Conductance $G_{\text {NS }}$ velsus Feimt energy of a quantum point contact between a nor mal and a superconducting reservorr (shown schematically in the inset) The dotted curve is twice the conductance $G_{\mathrm{N}}$ for the case of two normal reservous [20] The constuction is defined by the 2D saddle point potentral $V(x y)=V_{0}-\frac{1}{2} m \omega_{x}^{2} x^{2}+\frac{1}{2} m \omega_{y}^{2} y^{2}$ with $\omega_{y} / \omega_{x}=3 G_{N S}$ is calculated from eq (2 22) with $T_{n}=\left[1+\exp \left(-2 \pi \varepsilon_{n} / \hbar \omega_{x}\right)\right]{ }^{1} \varepsilon_{n} \equiv E_{\Gamma}-V_{0}-\left(n-\frac{1}{2}\right) \hbar \omega_{y}$ (From 1ef $[15])$

tiansition iegion, where $G_{\mathrm{NS}}$ lies below twice $G_{\mathrm{N}}$ This is actually a 11 gorous inequality, which follows from eqs (2 22) and (2 23) for arbutrary transmission matrix

$$
G_{\mathrm{NS}} \leq 2 G_{\mathrm{N}}, \forall t
$$

\section{Quantum dot}

Consider next a small confined region (of dimensions comparable to the Fermı wavelength), which is weakly coupled by tunnel barriers to two electron iesei vorrs We assume that transport through this quantum dot occurs via resonant tunneling through a single bound state Let $\varepsilon_{\text {res }}$ be the energy of the resonant level, relative to the Fermı level in the reservorrs, and let $\gamma_{1} / \hbar$ and $\gamma_{2} / \hbar$ be the tunnel 1 ates through the two barrers We denote $\gamma \equiv \gamma_{1}+\gamma_{2}$ If $\gamma \ll \Delta E$ (with $\Delta E$ the level spacing in the quantum dot), the conductance $G_{\mathrm{N}}$ in the case of 


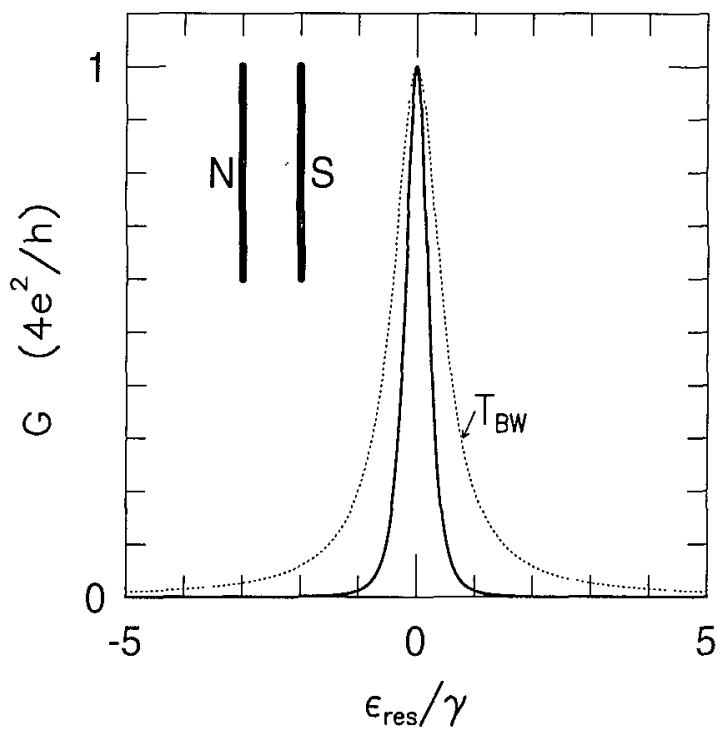

Fig. 4. Conductance versus energy of the resonant level, from eq. (3.8) for the case of equal tunnel barriers (solid curve). The dotted curve is the Breit-Wigner transmission probability (3.4). The inset shows schematically the normal-metal - quantum-dot - superconductor junction.

non-interacting electrons has the form

$$
\frac{h}{2 e^{2}} G_{\mathrm{N}}=\frac{\gamma_{1} \gamma_{2}}{\varepsilon_{\mathrm{res}}^{2}+\frac{1}{4} \gamma^{2}} \equiv T_{\mathrm{BW}}
$$

with $T_{\mathrm{BW}}$ the Breit-Wigner transmission probability at the Fermi level. The normal-state transmission matrix $t_{12}(\varepsilon)$ which yields this conductance has matrix elements [21]

$$
t_{12}(\varepsilon)=U_{1} \tau(\varepsilon) U_{2}, \tau(\varepsilon)_{n m} \equiv \frac{\sqrt{\gamma_{1 n} \gamma_{2 m}}}{\varepsilon-\varepsilon_{\mathrm{res}}+\frac{1}{2} \mathrm{i} \gamma},
$$

where $\sum_{n} \gamma_{1 n} \equiv \gamma_{1}, \sum_{n} \gamma_{2 n} \equiv \gamma_{2}$, and $U_{1}, U_{2}$ are two unitary matrices (which need not be further specified).

Let us now investigate how the conductance (3.4) is modified if one of the two reservoirs is in the superconducting state. The transmission matrix product $t_{12} t_{12}^{\dagger}$ (evaluated at the Fermi level $\varepsilon=0$ ) following from eq. (3.5) is

$$
t_{12} t_{12}^{\dagger}=U_{1} M U_{1}^{\dagger}, M_{n m} \equiv \frac{T_{\mathrm{BW}}}{\gamma_{1}} \sqrt{\gamma_{1 n} \gamma_{1 m}}
$$


Its e1genvalues are

$$
T_{n}= \begin{cases}T_{\mathrm{BW}} & \text { if } n=1 \\ 0 & \text { if } 2 \leq n \leq N\end{cases}
$$

Substitution into eq (22) yields the conductance

$$
G_{\mathrm{NS}}=\frac{4 e^{2}}{h}\left(\frac{T_{\mathrm{BW}}}{2-T_{\mathrm{BW}}}\right)^{2}=\frac{4 e^{2}}{h}\left(\frac{2 \gamma_{1} \gamma_{2}}{4 \varepsilon_{1 \mathrm{es}}^{2}+\gamma_{1}^{2}+\gamma_{2}^{2}}\right)^{2}
$$

The conductance on resonance $\left(\varepsilon_{1 \mathrm{es}}=0\right)$ is maximal in the case of equal tunnel rates $\left(\gamma_{1}=\gamma_{2}\right)$, and is then equal to $4 e^{2} / h-$ independent of $\gamma$ The lineshape for this case is shown in fig 4 (solid curve) It differs substantially from the Lolentzian lineshape (34) of the Breit-Wigner formula (dotted curve)

The amplitude and lineshape of the conductance resonance (38) does not depend on the relative magnitude of the resonance width $\gamma$ and the superconducting energy gap $\Delta_{0}$ This is in contrast to the supercurient resonance in a superconductor - quantum dot — superconductor Josephson junction, which depends sensitively on the ratio $\gamma / \Delta_{0}[22,23]$ The difference can be traced to the fact that the conductance (in the zero-temperature, zeio-voltage limit) is strictly a Fermilevel property, whereas all states within $\Delta_{0}$ of the Fermi level contribute to the Josephson effect (For an extension of eq (38) to finite voltages, see ref [24]) Since we have assumed non-interacting quasıpartıcles, the above results apply to a quantum dot with a small charging energy $U$ for double occupancy of the iesonant state Devyatov and Kupriyanov [25], and Hekkıng et al [26], have studied the influence of Coulomb repulsion on resonant tunneling through an NS junction, in the temperature regime $k_{\mathrm{B}} T \gg \gamma$ where the resonance is thermally broadened The extension to the low-temperature regime of an intrinsically bioadened resonance remains to be investigated

\section{Disordered Junction}

We now tuin to the regime of diffusive ti ansport through a disordered point contact or microbridge between a normal and a superconductıng ieseivoir The model consideied is that of an NS junction containing a disordered normal iegion of length $L$ much greater than the mean free path $l$ for elastic impurity scattering, but much smaller than the localızation length $N l$ We calculate the average conductance of the junction, averaged over an ensemble of impurity configurations We begin by parameterizing the transmission eigenvalue $T_{n}$ in terms of a channeldependent localization length $\zeta_{n}$

$$
T_{n}=\frac{1}{\cosh ^{2}\left(L / \zeta_{n}\right)}
$$


A fundamental result in quantum transport is that the inverse localization length is uniformly distributed between 0 and $1 / \zeta_{\mathrm{min}} \simeq 1 / l$ for $l \ll L \ll N l$ [27-30] One can therefore write

$$
\frac{\left\langle\sum_{n=1}^{N} f\left(T_{n}\right)\right\rangle}{\left\langle\sum_{n=1}^{N} T_{n}\right\rangle}=\frac{\int_{0}^{L / \zeta_{\min }} \mathrm{d} x f\left(\cosh ^{-2} x\right)}{\int_{0}^{L / \zeta_{\min }} \mathrm{d} x \cosh ^{-2} x}=\int_{0}^{\infty} \mathrm{d} x f\left(\cosh ^{-2} x\right),
$$

where $\langle>$ indicates the ensemble average and $f(T)$ is an arbitrary function of the transmission eigenvalue such that $f(T) \rightarrow 0$ for $T \rightarrow 0$ In the second equalıty in eq $(310)$ we have used that $L / \zeta_{\mathrm{mnn}} \simeq L / l \gg 1$ to replace the upper integration limit by $\infty$

Combinıng eqs (2 22), (2 23), and (3 10), we find

$$
\left\langle G_{\mathrm{NS}}\right\rangle=2\left\langle G_{\mathrm{N}}\right\rangle \int_{0}^{\infty} \mathrm{d} x\left(\frac{\cosh ^{-2} x}{2-\cosh ^{-2} x}\right)^{2}=\left\langle G_{\mathrm{N}}\right\rangle
$$

We conclude that - although $G_{\mathrm{NS}}$ according to eq (222) is of second order in the transmission eigenvalues $T_{n}$ - the ensemble aveiage $\left\langle G_{\mathrm{NS}}\right\rangle$ is of first ouder in $l / L$ The resolution of this paradox is that the T's are not distibuted uniformly, but are either exponentially small (closed channels) or of order unity (open channels) [28] Hence the average of $T_{n}^{2}$ is of the same order as the average of $T_{n}$ Off-diagonal elements of the transmission matrix $t t^{\dagger}$ are crucial to arrive at the result (3 11) Indeed, if one would evaluate eq . (22) with the tiansmission eigenvalues $T_{n}$ ieplaced by the modal transmission probabilities $\mathcal{T}_{n}$, one would find a totally wrong result Since $\mathcal{T}_{n} \simeq l / L \ll 1$, one would find $G_{\mathrm{NS}} \simeq$ $(l / L) G_{\mathrm{N}}$ - which underestimates the conductance of the NS junction by the factor $L / l$

Previous work $[31,32]$ had obtaned the equality of $G_{\mathrm{NS}}$ and $G_{\mathrm{N}}$ tiom semtclassical equations of motion, as was appropriate for macroscopic systems which are large compared to the normal-metal phase-coherence length $l_{\phi}$ The present deivation, in contrast, is fully quantum mechanical It applies to the "mesoscopic" legime $L<l_{\phi}$, in which transport is phase coherent Takane and Ebisawa [33] have studied the conductance of a disordered phase-coherent NS junction by numerical simulation of a two-dimensional tight-binding model They tound $\left\langle G_{\mathrm{NS}}\right\rangle=\left\langle G_{\mathrm{N}}\right\rangle$ within numerical accuracy for $l \ll L \ll N l$, in agreement with eq (3 11)

If the condition $L \ll N l$ is relaxed, differences between $\left\langle G_{N S}\right\rangle$ and $\left\langle G_{N}\right\rangle$ appear To lowest order in $L / N l$, the difference is a manitestation of the weaklocalization effect, as we discuss in the following section 


\section{Weak localization}

An NS junction shows an enhanced weak-localization eftect, in comparison with the noimal state [15] The orlgin of the enhancement can be understood in a simple way, as follows

We ietuin to the parameterization $T_{n} \equiv 1 / \cosh ^{2}\left(L / \zeta_{n}\right)$ introduced in eq (39), and define the density of localization lengths $\rho(\zeta, L) \equiv\left\langle\sum_{n} \delta\left(\zeta-\zeta_{n}\right)\right\rangle_{L}$ The subscript $L$ iefers to the length of the disordered region Using the identity $\cosh 2 x=2 \cosh ^{2} x-1$, the ensemble-average of eq (222) becomes

$$
\left\langle G_{\mathrm{NS}}\right\rangle_{L}=\frac{4 e^{2}}{h} \int_{0}^{\infty} \mathrm{d} \zeta \rho(\zeta, L) \cosh ^{-2}(2 L / \zeta)
$$

In the same parameterization, one has

$$
\left\langle G_{\mathrm{N}}\right\rangle_{L}=\frac{2 e^{2}}{h} \int_{0}^{\infty} \mathrm{d} \zeta \rho(\zeta, L) \cosh ^{-2}(L / \zeta)
$$

In the "open-channel approximation" [34], the integrals over $\zeta$ are restricted to the range $\zeta>L$ of localization lengths greater than the length of the conductor In this range the density $\rho(\zeta, L)$ is approximately independent of $L$ The whole $L$-dependence of the integrands in eqs (41) and (42) lies then in the argument of the hyperbolic cosine, so that

$$
\left\langle G_{\mathrm{NS}}\right\rangle_{L}=2\left\langle G_{\mathrm{N}}\right\rangle_{2 L}
$$

This deiıvation formalizes the intuitive notion that Andieev 1eflection at an NS interface etfectively doubles the length of the normal-metal conductor [33]

Consider now the geometry $W \ll L$ relevant for a microbridge In the normal state one has

$$
\left\langle G_{\mathrm{N}}\right\rangle=(W / L) \sigma_{\text {Diude }}-\delta G_{\mathrm{N}},
$$

where $\sigma_{\text {Diude }}$ is the classical Drude conductivity The $L$-independent term $\delta G_{\mathrm{N}}$ is the weak-localization correction, given by [35] $\delta G_{N}=\frac{2}{3} e^{2} / h$ Equation (4 3) then implies that

$$
\left\langle G_{\mathrm{NS}}\right\rangle=(W / L) \sigma_{\mathrm{D} \text { ude }}-\delta G_{\mathrm{NS}},
$$

with $\delta G_{\mathrm{NS}}=2 \delta G_{\mathrm{N}}$ We conclude that Andieev reflection increases the weaklocalization coirection, by a factor of two according to this qualitative argument [15] A ingoious theory [36-38] of weak localization in an NS microbridge shows that the increase is actually somewhat less than a factor of two, ${ }^{*}$

$$
\delta G_{\mathrm{NS}}=\left(2-8 \pi^{-2}\right) e^{2} / h=178 \delta G_{\mathrm{N}}
$$

* Equation (46) follows fiom the general formula $\delta A=\frac{1}{4} a(1)+\int_{0}^{\infty} \mathrm{d} x\left(4 x^{2}+\right.$ $\left.\pi^{2}\right)^{-1} a\left(\cosh ^{-2} x\right)$ for the weak localization conection in a wile geometiy wheic $A$ is an atbit raty transport property of the form $A=\sum_{n} a\left(T_{n}\right)$ 
As pointed out in ref [39], the enhancement of weak localization in an NS Junction can be observed experimentally as a $d i p$ in the differential conductance $G_{\mathrm{NS}}(V)=\partial I / \partial V$ around zero voltage The dip occurs because an applied voltage destroys the enhancement of weak localization by Andreev reflection, thereby increasing the conductance by an amount

$$
\delta G_{\mathrm{NS}}-\delta G_{\mathrm{N}} \approx 05 e^{2} / h
$$

at zero temperature [At finite temperatures, we expect a reduction of the size of dip by a factor* $\left(L_{\mathrm{c}} / L\right)^{2}$, where $L_{\mathrm{c}}=\min \left(l_{\phi}, \sqrt{\hbar D / k_{\mathrm{B}} T}\right)$ is the length over which electrons and holes remain phase coherent] We emphasize that in the noimal state, weak localization can not be detected in the current-voltage characteristic The reason why a dip occurs in $G_{\mathrm{NS}}(V)$ and not in $G_{\mathrm{N}}(V)$ is that an applied voltage (in contrast to a magnetic field) does not break time-reversal symmetry - but only affects the phase coherence between the elections and the Andieev-reflected holes (which differ in energy by up to $2 \mathrm{eV}$ ) The width $V_{\mathrm{c}}$ of the conductance dip is of the ordel of the Thouless energy $E_{\mathrm{c}} \equiv \pi h D / L^{2}$ (with $D$ the diffusion coefficient of the junction, $L$ should be replaced by $L_{\mathrm{c}}$ if $L>L_{\mathrm{c}}$ ) This energy scale is such that an electron and a hole acquire a phase difference of order $\pi$ on traversing the junction The energy $E_{\mathrm{c}}$ is much smaller than the superconducting energy gap $\Delta_{0}$, provided $L \gg \xi\left(\right.$ with $\xi \simeq\left(\hbar D / \Delta_{\circ}\right)^{1 / 2}$ the superconducting coherence length in the dirty-metal limit) The separation of energy scales is important, in order to be able to distinguish experimentally the cuisent due to Andreev reflection below the energy gap from the quasi-particle current above the energy gap

The first measurement of the conductance dip predicted in ref [39] has been ieported recently by Lenssen et al [40] The system studied consists of the twodimensional electron gas in a GaAs/AlGaAs heterostructure with $\mathrm{Sn} / \mathrm{T}$ i superconducting contacts ( $W=10 \mu \mathrm{m}, L=08 \mu \mathrm{m}$ ) No supercurrent is observed, presumably because $l_{\phi} \simeq 04 \mu \mathrm{m}$ is smaller than $L$ (The phase-coherence length $l_{\phi}$ is estimated from a conventional weak-localization measurement in a magnetic field) The data for the differential conductance is reproduced in fig 5 At the lowest temperatures $(10 \mathrm{mK})$ a rather small and narrow conductance dip develops, superimposed on a large and broad conductance minimum The size of the conductance dip is about $2 e^{2} / h$ Since in the experimental geometry $W>L>l_{\phi}$, and there are two NS interfaces, we would expect a dip of or-

* The reduction factor $\left(L_{c} / L\right)^{2}$ for the size of the conductance dip when $W<L_{\mathrm{c}}<L$ is estumated as follows Consider the wire as consisting of $L / L_{\mathrm{c}}$ phase-coherent segments of length $L_{\mathrm{c}}$ in sertes The fit st segment, adjacent to the supetconductor, has a conductance $\operatorname{dip} \delta G_{1} \simeq e^{2} / h$, while the other segments have no conductance dip The resistance $R_{1}$ of a single segment is a fraction $L_{\mathrm{c}} / L$ of the total resistance $R$ of the wne Since $\delta G / G=-\delta R / R=-\delta R_{1} / R$ and $\delta R_{1}=-R_{1}^{2} \delta G_{1} \simeq$ $-\left(L_{\mathrm{c}} / L\right)^{2} R^{2} e^{2} / h$ we find $\delta G \simeq\left(L_{\mathrm{c}} / L\right)^{2} e^{2} / h$ 


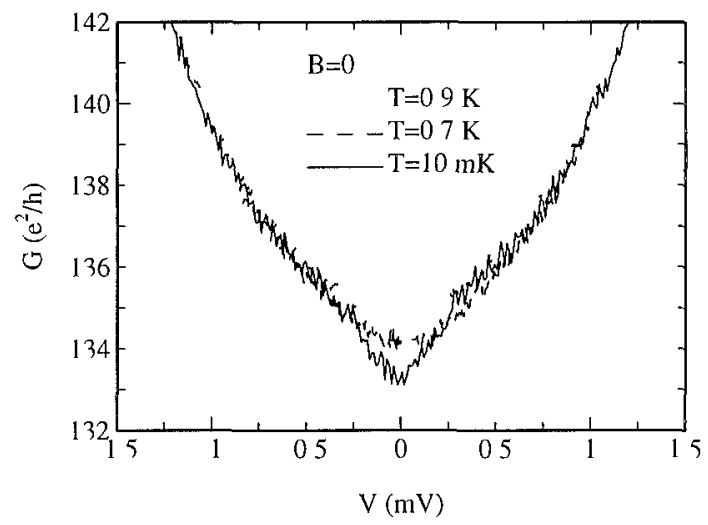

Fig 5 Differential conductance as a function of applied voltage at thee different temperatures Experimental data by Lenssen et al fot a two dimensional election gas with supeiconductng contacts The dip around zero voltage which is superimposed on the broad minimum at the lowest temperature is attubuted to the enhancement of weak localization by Andieev reflection (F1om ref [40])

der $2\left(W / l_{\phi}\right)\left(l_{\phi} / L\right)^{2} \times 05 e^{2} / h \simeq 6 e^{2} / h$, simply by countıng the number of phase coherent segments adjacent to the superconductor This is thiee times as latge as obseived, but the presence of a tunnel barrier at the NS interface might easily account for this discrepancy (The Schottky barrier at the interface between a semiconductor and superconductor presents a natural origin for such a barrier) The conductance dip has width $V_{\mathrm{c}} \simeq 025 \mathrm{mV}$, which is less than the energy gap $\Delta_{o}=056 \mathrm{meV}$ of bulk $\mathrm{Sn}$ - but not by much Experiments with a larger sepat ation of energy scales are required for a completely unambiguous identification of the phenomenon

An essential requirement for the appearance of a dip in the differential conductance is a high probability for Andreev reflection at the NS boundary This is illustrated in fig 6, which shows the results of numerical simulations [39] of tiansport through a disordered normal region connected via a tunnel barner to a superconductor The tunnel barrier is characterized by a transmission probability per mode $\Gamma$ The dash dotted lines refer to an ideal interface $(\Gamma=1)$, and show the conductance $d i p$ due to weak localization, discussed above For $\Gamma \simeq 02$ 04 the data for $G_{\mathrm{NS}}$ (filled circles) shows a crossover* to a conductance peak This is the phenomenon of reflectionless tunneling, discussed in the following section

* The crossover is accompanied by an overshoot around $\mathrm{eV} \approx L_{\mathrm{c}}$ indicating the absence of an excess current (1 e the lineat $I-V$ chatactenstic for $\mathrm{eV} \gg L_{\mathrm{c}}$ extrapolates back through the outgin) We do not have an analytical explanation for the oveishoot 


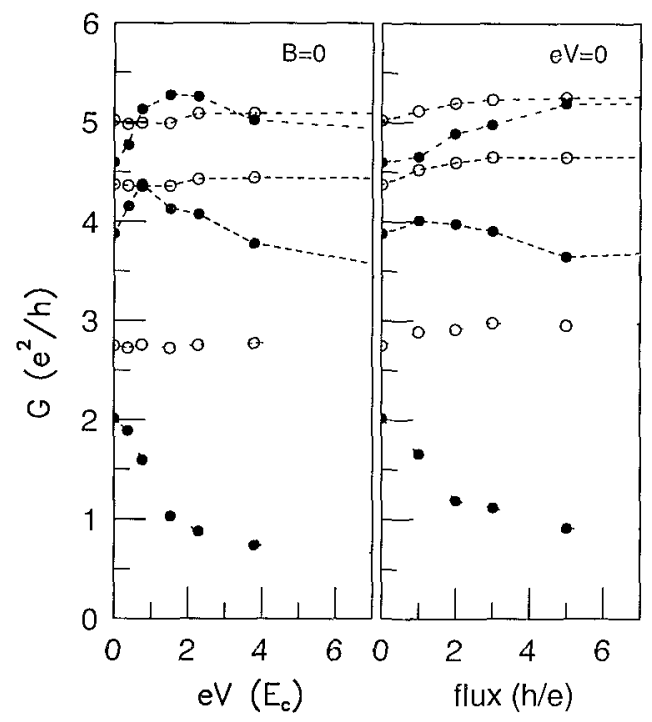

Fig 6 Voltage and magnettc field dependence of $G_{N S}$ (filled crrcles) and $G_{N}$ (open cucles) Numerical simulation of a disordered normal region $(L / W=48, l / L=012, N=11)$ in seives with a tunnel banter (transmission probability per mode $\Gamma$, dotted lines $\Gamma=02$, dashed $\Gamma=06$, dash-dotted $\Gamma=1$ ) Note the crossove1 tiom a $d i p$ (weak localization) to a peak (1eflectionless tunneling) in $G_{N S}$ on teducing $\Gamma$ (Fiom ref [39])

\section{Reflectionless tunneling}

In 1991, Kastalsky et al. [41] discovered a large and narow peak in the difterential conductance of a $\mathrm{Nb}$-InGaAs junction. We reproduce thell data in fig. 7. (A similar peak is observed as a function of magnetic field.) Since then a gieat deal of experimental [42-48], numerical [39,49], and analytical work [50-54] has been done on this effect. Here we focus on the explanation in terms of disorderinduced opening of tunneling channels [30,54], which is the most natural from the view point of the scattering formula (222), and which we feel captures the essence of the effect. Equivalently, the conductance peak can be explained in terms of a non-equilibrium proximity effect, which is the preferred explanation in a Green's function formulation of the problem [52,55-57]. We begin by reviewing the numencal work [39].

\subsection{Numerical sumulations}

A sharp peak in the conductance around $V, B=0$ is evident in the numerical simulations for $\Gamma=0.2$ (dotted lines in fig. 6). While $G_{\mathrm{N}}$ depends only weakly on $B$ 


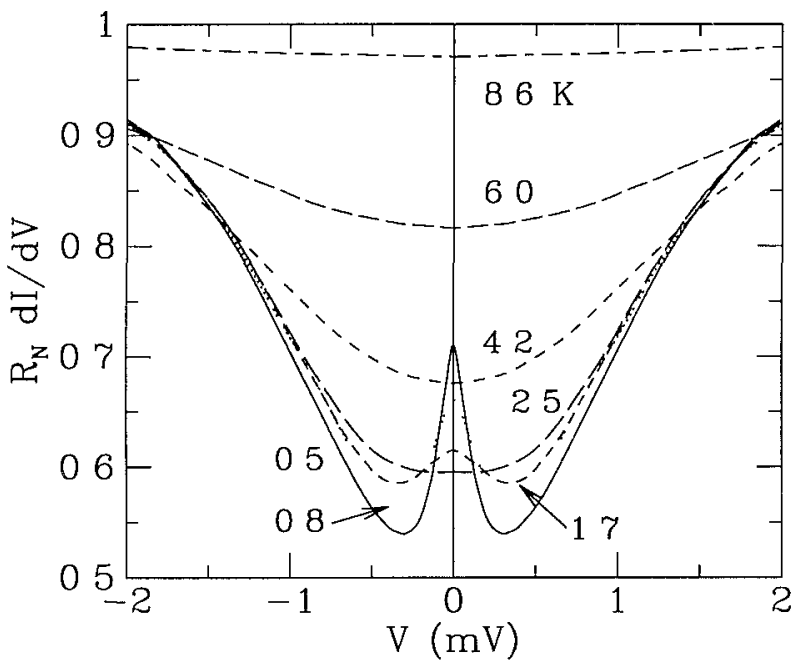

Fig 7 Diffeiential conductance (normalized by the normal-state 1esistance $R_{N}=027 \Omega$ ) as a function of applicd voltage at seven different temperatures Experimental data by Kastalsky et al for a Nb-InGaAs junction Note the difference with fig 5 A peak rather than a dip develops at the lowest temperatures and the size of the peak $\left(06 \Omega^{-1} \simeq 15 \times 10^{4} e^{2} / h\right)$ is toun ordes of magnitude gieate The width of the peak 15 comparable to the width of the dip in fig 5 (From 1ef [41])

and $V$ in this range (open circles), $G_{N S}$ drops abruptly (filled circles) The width of the conductance peak in $B$ and $\mathrm{eV}$ is respectively of order $B_{\mathrm{c}}=h / e L W$ (one flux quantum through the normal 1egion) and $e V_{\mathrm{c}}=\pi \hbar D / L^{2} \equiv E_{\mathrm{c}}$ (the Thouless energy) The width of the peak is the same as the width of the conductance dip due to weak localization, which occurs tor larger barriet tiansparencies The size of the peak is much greater than the dip, however

It is instiuctive to first discuss the classical resistance $R_{\mathrm{NS}}^{\text {class }}$ of the NS junction The basic approximation in $R_{\mathrm{NS}}^{\text {class }}$ is that currents rather than amplitudes ate matched at the NS interface [31] The result is

$$
R_{\mathrm{NS}}^{\text {class }}=\left(h / 2 N e^{2}\right)\left[L / l+2 \Gamma^{-2}+\mathcal{O}(1)\right]
$$

The contıbution fiom the barrier is $\propto \Gamma^{-2}$ because tunneling into a supeiconductor is a two-particle process [58] Both the incident electron and the Andieevreflected hole have to tunnel thiough the barrier (the net result being the addition of a Cooper pair to the superconductıng condensate [1]) Equatıon (5 1) is to be contrasted with the classical resistance $R_{\mathrm{N}}^{\text {class }}$ in the noimal state,

$$
R_{\mathrm{N}}^{\text {class }}=\left(h / 2 N e^{2}\right)\left[L / l+\Gamma^{-1}+\mathcal{O}(1)\right],
$$

where the contribution of a resistive barrier is $\propto \Gamma^{-1}$ In the absence of a tunnel 


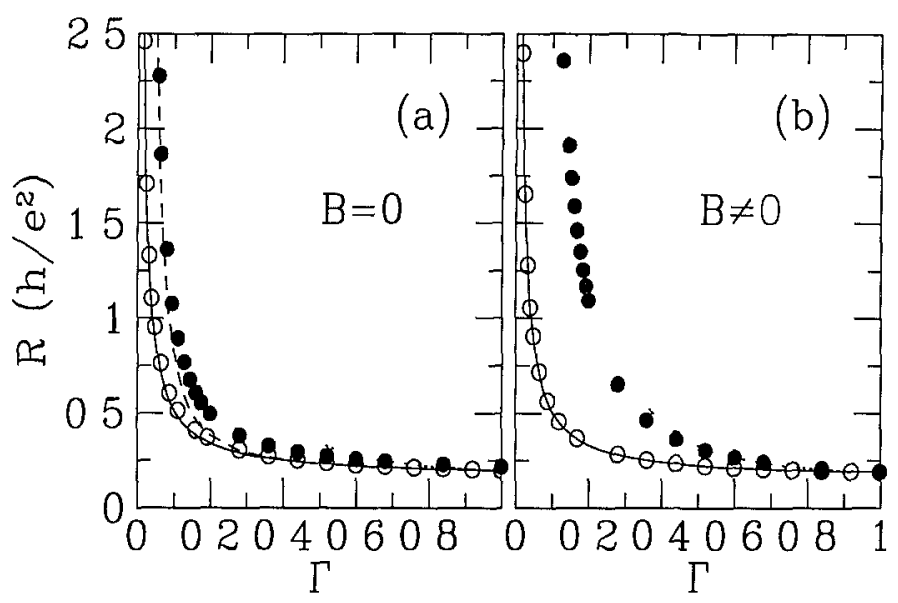

Fig 8 Filled circles Numerically calculated resistance $R_{\text {NS }}$ of a disordered NS junction versus the u ansmission probability per mode $\Gamma$ of the tunnel barrier at the NS interface Open cucles Resistance $R_{\mathrm{N}}$ of the same junction in the normal state (a) is for zero magnetic field (b) is for a flux of $10 \mathrm{~h} / \mathrm{e}$ through the disordered region The dotted and solid curves are the classical eqs $(51)$ and $(52)$ The dashed curve is the theory of ref [52] which for $\Gamma \gg l / L \approx 012$ concides with eq (53) (From Ief [39])

barrier ( 1 e for $\Gamma=1), R_{\mathrm{NS}}^{\text {class }}=R_{\mathrm{N}}^{\text {class }}$ for $L \gg l$, 1n agreement with refs $[31,32]$ Let us now see how these classical results compare with the simulations [39]

In fig 8 we show the resistance (at $V=0$ ) as a function of $\Gamma$ in the absence and presence of a magnetic field (The parametess of the disordered region are the same as for fig 6 ) There is good agreement with the classical eqs (51) and (52) for a magnetic field corresponding to 10 flux quanta through the disordered segment (fig 8b) For $B=0$, however, the situation is different (fig 8a) The normal-state resistance (open circles) still follows approximately the classical formula (solid curve) (Deviations due to weak localization are noticeable, but small on the scale of the figure) In contrast, the resistance of the NS junction (filled circles) hies much below the classical prediction (dotted curve) The numerical data shows that for $\Gamma \gg l / L$ one has approximately

$$
R_{\mathrm{NS}}(B=0, V=0) \approx R_{\mathrm{N}}^{\text {class }},
$$

which for $\Gamma \ll 1$ is much smaller than $R_{\mathrm{NS}}^{\text {class }}$ This is the phenomenon of reflectoonless tunneling In fig $8 \mathrm{a}$ the barrier contributes to $R_{\mathrm{NS}}$ in order $\Gamma^{-1}$, just as for single-particle tunneling, and not in order $\Gamma^{-2}$, as expected for two-particle tunneling It is as if the Andreev-reflected hole is not reflected by the barrier The interfering trajectories responsible for this effect were first identıfied by Van Wees 
et al [50] The numerical data of fig 8a is in good agreement with the Green's function calculation of Volkov, Zautsev, and Klapwıjk [52] (dashed curve) Both these papers have played a crucral role in the understanding of the effect The scaling theory reviewed below [54] is essentially equivalent to the Green's function calculation, but has the advantage of explicitly demonstratıng how the opening of tunneling channels on increasing the length $L$ of the disordered region induces a transition from a $\Gamma^{-2}$ dependence to a $\Gamma^{-1}$ dependence when $L \simeq l / \Gamma$

\section{Scaling theory}

We use the parameterization

$$
T_{n}=\frac{1}{\cosh ^{2} x_{n}}
$$

similar to eq (39), but now with a dimensionless variable $x_{n} \in[0, \infty)$ The density of the $x$-variables, for a length $L$ of disordered region, is denoted by

$$
\rho(x, L)=\left\langle\sum_{n} \delta\left(x-x_{n}\right)\right\rangle_{L}
$$

For $L=0,1$ e in the absence of disorder, we have the initial condition imposed by the barrier,

$$
\rho(x, 0)=N \delta\left(x-x_{0}\right),
$$

with $\Gamma=1 / \cosh ^{2} x_{0}$ The scalıng theory describes how $\rho(x, L)$ evolves with increasing $L$ This evolution is governed by the equation

$$
\frac{\partial}{\partial s} \rho(x, s)=-\frac{1}{2 N} \frac{\partial}{\partial x} \rho(x, s) \frac{\partial}{\partial x} \int_{0}^{\infty} \mathrm{d} x^{\prime} \rho\left(x^{\prime}, s\right) \ln \left|\sinh ^{2} x-\sinh ^{2} x^{\prime}\right|,
$$

where we have defined $s \equiv L / l$ This non-linear diffusion equation was derived by Mello and Pichard [59] fiom a Fokker-Planck equation [34,60,61] for the joint distıbution function of all $N$ eigenvalues, by integratıng out $N-1$ eigenvalues and takıng the large- $N$ limit This limit iestricts its validity to the metallic regime $(N \gg L / l)$, and is sufficient to determine the leading order contribution to the average conductance, which is $\mathcal{O}(N)$ The weak-localization correction, which is $\mathcal{O}(1)$, is neglected here A prorı, eq (5 7) holds only for a "quasione-dimensional" wire geometry (length $L$ much greater than width $W$ ), because the Fokke1-Planck equation trom which it is derived requires $L \gg W$ Nume1ical simulations indicate that the geometry dependence only appears in the $\mathcal{O}(1)$ corrections, and that the $\mathcal{O}(N)$ contubutions are essentrally the same for a wire, square, or cube

In ref [54] it is shown how the scalıng equation (57) can be solved exactly, for arbitraty initial condition $\rho(x, 0) \equiv \rho_{0}(x)$ The method of solution is based 


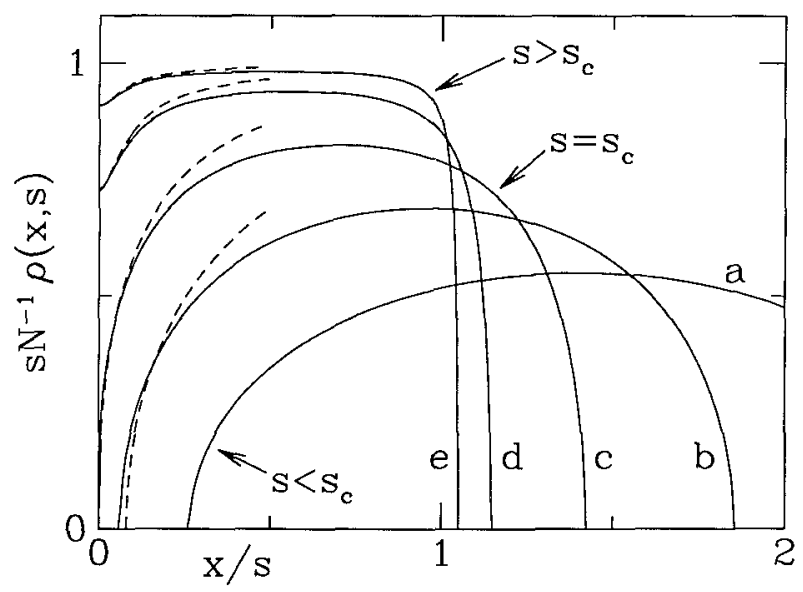

Fig 9 Engenvalue density $\rho(x, s)$ as a function of $x$ (1n units of $s=L / l$ ) for $\Gamma=01$ Curves a,b,c,d,e are for $s=2,4,9,30,100$, respectively The solid curves are from eq $(58)$, the dashed curves from eq (512) The collision of the density profile with the boundary at $x=0$, for $s=$ $s_{\mathrm{c}}=(1-\Gamma) / \Gamma$, signals the disorder-induced opening of tunnelung channels responsible for the reflectionless tunneling effect (Fiom ref [54])

on a mapping of eq. (57) onto Euler's equation for the 1sobaric flow of a twodimensional ideal fluid $L$ corresponds to time and $\rho$ to the $y$-component of the velocity field on the $x$-axis. [Please note that in this section $x$ is the auxiliary variable defined in eq. (5 4) and not the physical coordinate in fig. 2] The result is

$$
\rho(x, s)=(2 N / \pi) \operatorname{Im} U\left(x-10^{+}, s\right),
$$

where the complex function $U(z, s)$ is determined by

$$
U(z, s)=U_{0}(z-s U(z, s)) .
$$

The function $U_{0}(z)$ is fixed by the initial condition,

$$
U_{0}(z)=\frac{\sinh 2 z}{2 N} \int_{0}^{\infty} \mathrm{d} x^{\prime} \frac{\rho_{0}\left(x^{\prime}\right)}{\sinh ^{2} z-\sinh ^{2} x^{\prime}} .
$$

The implicit equation (5.9) has multiple solutions in the entire complex plane; We need the solution for which both $z$ and $z-s U(z, s)$ lie in the strip between the lines $y=0$ and $y=-\pi / 2$, where $z=x+\mathrm{i} y$.

The initial condition (5.6) corresponds to

$$
U_{0}(z)=\frac{1}{2} \sinh 2 z\left(\cosh ^{2} z-\Gamma^{-1}\right)^{-1}
$$


The resulting density (58) is plotted in fig 9 (solid curves), for $\Gamma=01$ and several values of $s$ For $s \gg 1$ and $x \ll s$ it simplifies to

$$
\begin{aligned}
& x=\frac{1}{2} \operatorname{arccosh} \tau-\frac{1}{2} \Gamma s\left(\tau^{2}-1\right)^{1 / 2} \cos \sigma \\
& \sigma \equiv \pi s N^{-1} \rho(x, s), \tau \equiv \sigma(\Gamma s \sin \sigma)^{-1}
\end{aligned}
$$

shown dashed in fig 9 Equation (5 12) agrees with the esult of a Gieen's function calculation by Nazarov [30] For $s=0$ (no disordel), $\rho$ is a delta tunction at $x_{0}$ On adding disorder the eigenvalue density iapidly spieads along the $x$-axis (cuive a), such that $\rho \leq N / s$ for $s>0$ The sharp edges of the density profile, so uncharacteristic for a dittusion profile, reveal the hydıdynamic nature of the scaling equation (57) The upper edge is at

$$
x_{\max }=s+\frac{1}{2} \ln (s / \Gamma)+\mathcal{O}(1)
$$

Since $L / x$ has the physical significance of a localization length [34], this upper edge corresponds to a minimum localization length $\xi_{\mathrm{min}}=L / x_{\mathrm{m} \text { ?x }}$ of otder $l$ The lower edge at $x_{\mathrm{mm}}$ propagates from $x_{0}$ to 0 in a "tıme" $s_{\mathrm{c}}=(1-\Gamma) / \Gamma$ For $1 \ll s \leq s_{\mathrm{C}}$ one has

$$
x_{\min }=\frac{1}{2} \operatorname{arccosh}\left(s_{\mathrm{c}} / s\right)-\frac{1}{2}\left[1-\left(s / s_{\mathrm{c}}\right)^{2}\right]^{1 / 2}
$$

It follows that the maximum localization length $\xi_{\max }=L / x_{\min }$ increases if disorder is added to a tunnel junction This paradoxical iesult, that disordei enhances tiansmission, becomes intuitively obvious fiom the hydrodynamic cortespondence, which implies that $\rho(x, s)$ spreads both to larger and smaller $x$ as the fictitious time $s$ progresses When $s=s_{\mathrm{C}}$ the diffusion profile hits the boundary at $x=0$ (curve $\mathrm{c}$ ), so that $x_{\mathrm{m} 1 \mathrm{n}}=0$ This implies that for $s>s_{\mathrm{c}}$ there exist scattering states (eigentunctions of $t t^{\dagger}$ ) which tunnel thiough the barrier with near-unit transmission probability, even if $\Gamma \ll 1$ The number $N_{\text {open }}$ of transmission eigenvalues close to one (open channels) is of the order of the number of $x_{n}$ 's in the range 0 to 1 (since $T_{n} \equiv 1 / \cosh ^{2} x_{n}$ vanishes exponentially if $x_{n}>1$ ) Fol $s \gg s_{\mathrm{c}}$ (curve e) we estimate

$$
N_{\text {open }} \simeq \rho(0, s)=N\left(s+\Gamma^{-1}\right)^{-1},
$$

where we have used eq (5 12) The disorder induced opening of tunneling channels was discovered by Nazarov [30] It is the fundamental mechanism for the $\Gamma^{-2}$ to $\Gamma^{-1}$ transition in the conductance of an NS junction, as we now discuss

Accotding to eqs (2 22), (2 23), (5 4), and (5 5), the avelage conductances $\left\langle G_{\mathrm{NS}}\right\rangle$ and $\left\langle G_{\mathrm{N}}\right\rangle$ are given by the integrals

$$
\begin{aligned}
\left\langle G_{\mathrm{NS}}\right\rangle & =\frac{4 e^{2}}{h} \int_{0}^{\infty} \mathrm{d} x \rho(x, s) \cosh ^{-2} 2 x, \\
\left\langle G_{\mathrm{N}}\right\rangle & =\frac{2 e^{2}}{h} \int_{0}^{\infty} \mathrm{d} x \rho(x, s) \cosh ^{-2} x
\end{aligned}
$$


Here we have used the same trigonometric identity as in eq (4 1) For $\Gamma \gg l / L$ one is in the regime $s \gg s_{\mathrm{c}}$ of curve e in fig 9 Then the dominant contribution to the integrals comes from the range $x / s \ll 1$ where $\rho(x, s) \approx \rho(0, s)=N(s+$ $\left.\Gamma^{-1}\right)^{-1}$ is approximately independent of $x$ Substitution of $\rho(x, s)$ by $\rho(0, s)$ in eqs (5 16) and (5 17) yrelds directly

$$
\left\langle G_{\mathrm{NS}}\right\rangle \approx\left\langle G_{\mathrm{N}}\right\rangle \approx 1 / R_{\mathrm{N}}^{\text {class }},
$$

in agreement with the result (53) of the numerical simulations

Equation (5 18) has the linear $\Gamma$ dependence characteristic for reflectionless tunneling The crossover to the quadratic $\Gamma$ dependence when $\Gamma \lesssim l / L$ is obtained by evaluating the integrals $(516)$ and $(517)$ with the density $\rho(x, s)$ given by eq (5 8) The result is [54]

$$
\begin{aligned}
\left\langle G_{\mathrm{NS}}\right\rangle & =\left(2 N e^{2} / h\right)\left(s+Q^{-1}\right)^{-1}, \\
\left\langle G_{\mathrm{N}}\right\rangle & =\left(2 N e^{2} / h\right)\left(s+\Gamma^{-1}\right)^{-1}
\end{aligned}
$$

The "effective" tunnel probability $Q$ is defined by

$$
Q=\frac{\theta}{s \cos \theta}\left(\frac{\theta}{\Gamma s \cos \theta}(1+\sin \theta)-1\right),
$$

where $\theta \in(0, \pi / 2)$ is the solution of the transcendental equation

$$
\theta\left[1-\frac{1}{2} \Gamma(1-\sin \theta)\right]=\Gamma s \cos \theta
$$

For $\Gamma \ll 1$ (or $s \gg 1$ ) eqs (5 21) and (5 22) simplify to $Q=\Gamma \sin \theta$, $\theta=\Gamma s \cos \theta$, in precise agreement with the Green's function calculation of Volkov, Zătsev, and Klapwijk [52] Accordıng to eq (5 20), the normal state resistance increases linearly with the length $L$ of the disordered region, as expected from Ohm's law This classical reasoning fails if one of the contacts is in the superconductung state The scaling of the resistance $R_{\mathrm{NS}} \equiv 1 /\left\langle G_{\mathrm{NS}}\right\rangle$ with length, computed from eq (5 19), is plotted in fig 10 For $\Gamma=1$ the resistance increases monotonically with $L$ The ballistic limit $L \rightarrow 0$ equals $h / 4 N e^{2}$, half the contact resistance of a normal junction because of Andreev reflection (cf section 3 1) For $\Gamma \lesssim 05$ a resistance minımum develops, somewhat below $L=l / \Gamma$ The resistance minimum is associated with the crossover from a quadratic to a linear dependence of $R_{\mathrm{NS}}$ on $1 / \Gamma$

If $\Gamma s \gg 1$ one has $\theta \rightarrow \pi / 2$, hence $Q \rightarrow \Gamma$ In the opposite regime $\Gamma s \ll 1$ one has $\theta \rightarrow \Gamma s$, hence $Q \rightarrow \Gamma^{2} s$ The corresponding asymptotic expressions for $\left\langle G_{\mathrm{NS}}\right\rangle$ are (assuming $\Gamma \ll 1$ and $s \gg 1$ )

$$
\begin{aligned}
& \left\langle G_{\mathrm{NS}}\right\rangle=\left(2 N e^{2} / h\right)\left(s+\Gamma^{-1}\right)^{-1}, \text { if } \Gamma s \gg 1, \\
& \left\langle G_{\mathrm{NS}}\right\rangle=\left(2 N e^{2} / h\right) \Gamma^{2} s, \text { if } \Gamma s \ll 1
\end{aligned}
$$




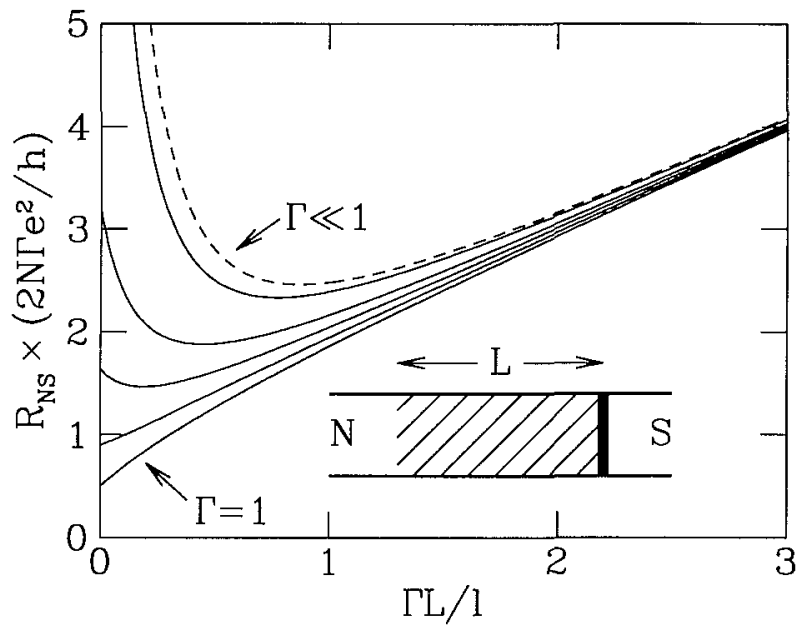

Fig 10 Dependence of the resistance $R_{\mathrm{NS}}$ on the length $L$ of the disordered normal region (hatched in the inset), for different values of the transmittance $\Gamma$ of the NS interface Solid curves are computed fiom eq (5 19), for $\Gamma=108,06,0401$ fiom bottom to top For $\Gamma \ll 1$ the dashed curve is appioached (Fiom ref [54])

In either limit the conductance is gieater than the classical result

$$
G_{\mathrm{NS}}^{\text {class }}=\left(2 N e^{2} / h\right)\left(s+2 \Gamma^{-2}\right)^{-1},
$$

which holds if phase coherence between electrons and holes is destroyed by a voltage or magnetic field The peak in the conductance around $V, B=0$ is of order $\Delta G_{\mathrm{NS}}=\left\langle G_{\mathrm{NS}}\right\rangle-G_{\mathrm{NS}}^{\text {class }}$, which has the relative magnitude

$$
\frac{\Delta G_{\mathrm{NS}}}{\left\langle G_{\mathrm{NS}}\right\rangle} \approx \frac{2}{2+\Gamma^{2} s}
$$

The scalıng theory assumes zero temperature Hekking and Nazarov [53] have studied the conductance of a resistive NS interface at finite temperatures, when $L$ is greater than the correlation length $L_{\mathrm{c}}=\min \left(l_{\phi}, \sqrt{\hbar D / k_{\mathrm{B}} T}\right)$ Their result is consistent with the limiting expression (524), if $s=L / l$ is ieplaced by $L_{c} / l$ The implication is that, if $L>L_{c}$, the non-linear scaling of the resistance shown in fig 10 only applies to a disordered segment of length $L_{\mathrm{c}}$ adjacent to the superconductor For the total resistance one should add the Ohmic contribution of order $\left(h / e^{2}\right)\left(L-L_{\mathrm{c}}\right) / l$ from the iest of the wire 


\section{Double-barrier Junction}

In the previous subsection we have discussed how the opening of tunneling channels ( 1 e the appearance of transmission eigenvalues close to one) by disorder leads to a minimum in the resistance when $L \simeq l / \Gamma$ The minımum separates a $\Gamma^{-1}$ trom a $\Gamma^{-2}$ dependence of the resistance on the transparency of the interface We tefeired to the $\Gamma^{-1}$ dependence as "reflectionless tunneling", since it is as if one of the two quasiparticles which form the Cooper pair can tunnel thiough the barı with probability one In the present subsection we will show, following ref [62], that a qualitatively similar effect occuis if the disorder in the normal iegion is ieplaced by a second tunnel baruer (tunnel piobability $\Gamma^{\prime}$ ) The iesistance at fixed $\Gamma$ shows a minimum as a function of $\Gamma^{\prime}$ when $\Gamma^{\prime} \simeq \Gamma$ For $\Gamma^{\prime} \lesssim \Gamma$ the resistance has a $\Gamma^{-1}$ dependence, so that we can speak again of reflectıonless tunneling

We considel an $\mathrm{NI}_{1} \mathrm{NI}_{2} \mathrm{~S}$ junction, where $\mathrm{N}=$ normal metal, $\mathrm{S}=$ superconductor, and $I_{2}=$ insulato or tunnel barrier (transmission probability per mode $\Gamma_{z} \equiv 1 / \cosh ^{2} \alpha_{2}$ ) We assume ballistic motion between the batrieis (The effect of disoider is discussed later) A straightforward calculation yields the transmission probabilities $T_{n}$ of the two barriers in series,

$$
\begin{aligned}
& T_{n}=\left(a+b \cos \varphi_{n}\right)^{-1}, \\
& a=\frac{1}{2}+\frac{1}{2} \cosh 2 \alpha_{1} \cosh 2 \alpha_{2}, \quad b=\frac{1}{2} \sinh 2 \alpha_{1} \sinh 2 \alpha_{2},
\end{aligned}
$$

where $\varphi_{n}$ is the phase accumulated between the barriers by mode $n$ Since the transmission matıx $t$ is diagonal, the transmission probabilities $T_{n}$ are identical to the eigenvalues of $t t^{\dagger}$ We assume that $L \gg \lambda_{\Gamma}\left(\lambda_{\Gamma}\right.$ is the Fermi wavelength) and $N \Gamma_{\imath} \gg 1$, so that the conductance is not dominated by a single iesonance In this case, the phases $\varphi_{n}$ are distributed uniformly in the interval $(0,2 \pi)$ and we may replace the sum over the transmission eigenvalues in eqs (2 22) and (2 23) by integrals over $\varphi \sum_{n=1}^{N} f\left(\varphi_{n}\right) \rightarrow(N / 2 \pi) \int_{0}^{2 \pi} \mathrm{d} \varphi f(\varphi)$ The result is

$$
\begin{aligned}
G_{\mathrm{NS}} & =\frac{4 N e^{2}}{h} \frac{\cosh 2 \alpha_{1} \cosh 2 \alpha_{2}}{\left(\cosh ^{2} 2 \alpha_{1}+\cosh ^{2} 2 \alpha_{2}-1\right)^{3 / 2}} \\
G_{N} & =\frac{4 N e^{2}}{h}\left(\cosh 2 \alpha_{1}+\cosh 2 \alpha_{2}\right)^{-1}
\end{aligned}
$$

These expressions are symmetıc in the indices 1 and 2 It does not mattel which of the two barrers is closest to the superconductor In the same way we can compute the entile distribution of the transmission eigenvalues, $\rho(T) \equiv \sum_{n} \delta(T-$ $\left.T_{n}\right) \rightarrow(N / 2 \pi) \int_{0}^{2 \pi} \mathrm{d} \varphi \delta(T-T(\varphi))$ Substituting $T(\varphi)=(a+b \cos \varphi)^{-1}$ from eq (527), one finds

$$
\rho(T)=\frac{N}{\pi T}\left(b^{2} T^{2}-(a T-1)^{2}\right)^{-1 / 2}
$$




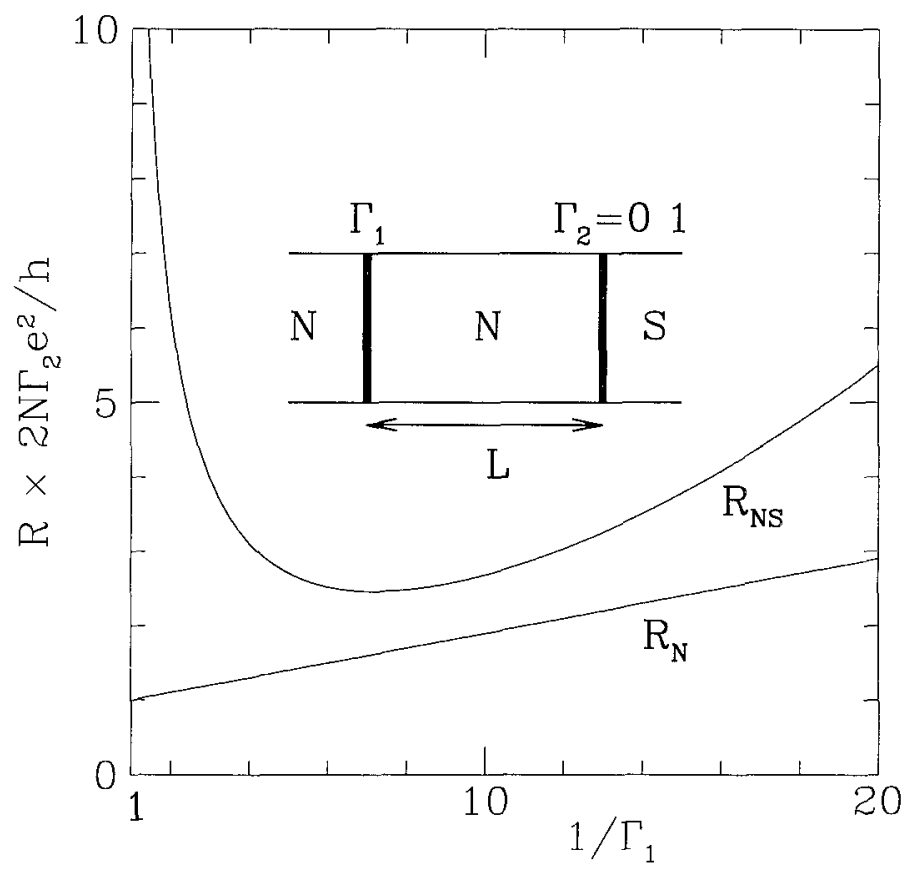

F1g 11 Dependence of the resistances $R_{\mathrm{N}}$ and $R_{\mathrm{NS}}$ of balhstic NININ and NINIS stuctures, respectively, on baries transparency $\Gamma_{1}$, while tiansparency $\Gamma_{2}=01$ is kept fixed [computed fiom eqs (529) and (5 30)] The inset shows the NINIS stucture considered (From ref [62])

In fig 11 we plot the resistance $R_{\mathrm{N}}=1 / G_{\mathrm{N}}$ and $R_{\mathrm{NS}}=1 / G_{\mathrm{NS}}$, following from eqs (5 29) and (5 30) Notice that $R_{\mathrm{N}}$ follows Ohm's law,

$$
R_{\mathrm{N}}=\frac{h}{2 N e^{2}}\left(1 / \Gamma_{1}+1 / \Gamma_{2}-1\right),
$$

as expected from classical considerations In contrast, the resistance $R_{\mathrm{NS}}$ has a minimum if one of the $\Gamma$ 's is varied while keeping the other fixed This resistance minımum cannot be explained by classical seires addition of barrier resistances If $\Gamma_{2} \ll 1$ is fixed and $\Gamma_{1}$ is varied, as in fig 11 , the minimum occurs when $\Gamma_{1}=\sqrt{2} \Gamma_{2}$ The minimal resistance $R_{\mathrm{NS}}^{\mathrm{min}}$ is of the same order of magnitude as the resistance $R_{\mathrm{N}}$ in the noimal state at the same value of $\Gamma_{1}$ and $\Gamma_{2}$ In paiticular, we find that $R_{\mathrm{NS}}^{\mathrm{min}}$ depends linearly on $1 / \Gamma_{\imath}$, whereas for a single barner $R_{\mathrm{NS}} \propto 1 / \Gamma^{2}$

The linear dependence on the barrier transparency shows the qualitative similarity of a ballistic NINIS junction to the disordered NIS junction considered in the previous subsection To illustrate the similarity, we compare in fig 12 the densit- 


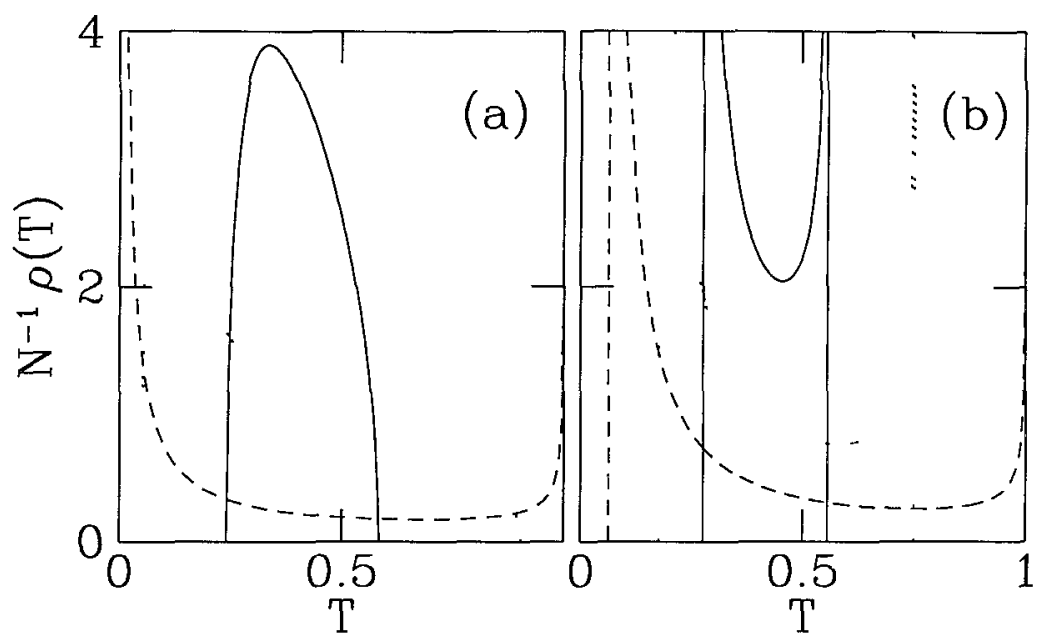

Fig 12 Density of transmission eigenvalues through a normal region contanning a potential batries (ttansmission probability $\mathrm{\Gamma}=04$ ) The left panel (a) shows the disorder induced opening of tunnel ung channels (solid curve $s=004$ dotted $s=04$, dashed $s=5$ where $s \equiv L / l$ ) The right panel (b) shows the opening of channels by a second tunnel banies (transparency $\Gamma^{\prime}$, solid curve $\Gamma^{\prime}=095$, dotted $\Gamma^{\prime}=08$, dashed $\Gamma^{\prime}=04$ ) The curves in (a) are computed from eq (58) the curves in (b) from eq (5 31) (From ref [62])

1es of normal-state transmission eigenvalues The left panel is for an NIS junction [computed using eq (5 8)], the right panel is for an NINIS junction [computed from eq (531)] In the NIS junction, disorder leads to a bimodal distribution $\rho(T)$, with a peak near zero transmission and another peak near unit transmisssion (dashed curve) A similar bimodal distribution appears in the ballistic NINIS junction, for approximately equal transmission probabilities of the two batrers There are also differences between the two cases The NIS junction has a unimodal $\rho(T)$ if $L / l<1 / \Gamma$, while the NINIS junction has a bimodal $\rho(T)$ for any ratio of $\Gamma_{1}$ and $\Gamma_{2}$ In both cases, the opening of tunneling channels, $1 \mathrm{e}$ the appearance of a peak in $\rho(T)$ near $T=1$, is the origin for the $1 / \Gamma$ dependence of the resistance

The scaling equation of section 52 can be used to investigate what happens to the resistance minımum if the region of length $L$ between the tunnel barriers contains impurities, with elastic mean free path $l$ As shown in ref [62], the resistance minimum persists as long as $l \gtrsim \Gamma L$ In the diffusive regime $(l \ll L)$ the scaling theory is found to agree with the Green's function calculation by Volkov, Zătsev, and Klapwıjk for a disordered NINIS junction [52] For stıong barriers 
$\left(\Gamma_{1}, \Gamma_{2} \ll 1\right)$ and strong disorder $(L \gg l)$, one has the two asymptotic formulas

$$
\begin{aligned}
G_{\mathrm{NS}} & =\frac{2 N e^{2}}{h} \frac{\Gamma_{1}^{2} \Gamma_{2}^{2}}{\left(\Gamma_{1}^{2}+\Gamma_{2}^{2}\right)^{3 / 2}}, \text { if } \Gamma_{1}, \Gamma_{2} \ll l / L, \\
G_{\mathrm{NS}} & =\frac{2 N e^{2}}{h}\left(L / l+1 / \Gamma_{1}+1 / \Gamma_{2}\right)^{-1}, \text { if } \Gamma_{1}, \Gamma_{2} \gg l / L
\end{aligned}
$$

Equation (5 33) coincides with eq (5 29) in the limit $\alpha_{1}, \alpha_{2} \gg 1$ (recall that $\Gamma_{\imath} \equiv$ $\left.1 / \cosh ^{2} \alpha_{2}\right)$ This shows that the eftect of disorder on the resistance minimum can be neglected as long as the resistance of the junction is dominated by the barriets In this case $G_{\mathrm{NS}}$ depends linearly on $\Gamma_{1}$ and $\Gamma_{2}$ only if $\Gamma_{1} \approx \Gamma_{2}$ Equation (5 34) shows that if the disorder dominates, $G_{\mathrm{NS}}$ has a linear $\Gamma$-dependence regardless of the relative magnitude of $\Gamma_{1}$ and $\Gamma_{2}$

We have assumed zero temperature, zero magnetıc field, and infinitesimal applied voltage Each of these quantities is capable of destroying the phase coherence between the electrons and the Andreev-reflected holes, which is responsible for the resistance minimum As far as the temperature $T$ and voltage $V$ are concerned, we require $k_{\mathrm{B}} T, e V \ll \hbar / \tau_{\text {dwell }}$ for the appearance of a resistance minımum, where $\tau_{\text {dwell }}$ is the dwell time of an electron in the region between the two barriers For a ballistic NINIS junction $\tau_{\text {dwell }} \simeq L / v_{\Gamma} \Gamma$, while for a disordered junction $\tau_{\text {dwell }} \simeq L^{2} / v_{\mathrm{F}} \Gamma l$ is larger by a factor $L / l$ It follows that the condition on temperature and voltage becomes more restrictive if the disorder increases, even if the resistance remains dominated by the barriers As far as the magnetic field $B$ is concerned, we require $B \ll h / e S$ (with $S$ the area of the junction perpendicular to $B$ ), if the motion between the barriers is diffusive For ballistic motion the trajectories enclose no flux, so no magnetic field dependence 1s expected

A possible experiment to verify these results might be scanning tunneling micioscopy (STM) of a metal particle on a superconducting substrate [63] The metal-superconductor interface has a fixed tunnel probability $\Gamma_{2}$ The probability $\Gamma_{1}$ for an electron to tunnel from STM to particle can be controlled by varying the distance (Volkov has recently analyzed this geometry in the iegime that the motion from STM to particle is diffusive rather than by tunneling [64] ) Anothes possibility is to create an NINIS junction using a two-dimensional electron gas in contact with a superconductor An adjustable tunnel barrier could then be implemented by means of a gate electrode

\section{Circuit theory}

The scalıng theory of ref [54], which was the subject of section 52 , descubes the transition from the ballistic to the diffusive regime In the diffusive regime it is equivalent to the Green's function theory of ref [52] A third, equivalent, 
theory for the diffusive regime was presented recently by Nazarov [65] Startıng from a continuity equation for the Keldysh Green's function [66], and applying the appropriate boundary conditions [67], Nazarov was able to formulate a set of rules which reduce the problem of computing the resistance of an NS junction to a simple exercise in circuit theory Furthermore, the approach can be applied without further complications to multi-termınal networks involving several normal and superconducting reservorrs Because of its practical importance, we discuss Nazarov's circuit theory in some detail

The supetconductors $S_{\imath}$ should all be at the same voltage, but may have a different phase $\phi_{2}$ of the pair potential Zero temperature is assumed, as well as infinitesimal voltage differences between the normal reservoirs (linear response) The reservoirs are connected by a set of diffusive normal-state conductors (length $L_{\imath}$, mean free path $l_{\imath}, s_{\imath} \equiv L_{\imath} / l_{\imath} \gg 1$ ) Between the conductors there may be tunnel barriers (tunnel probability $\Gamma_{2}$ ) The presence of superconducting reservoirs has no effect on the resistance $\left(h / 2 N e^{2}\right) s_{\imath}$ of the diffusive conductors, but affects only the resistance $h / 2 N e^{2} \Gamma_{\imath}^{\text {eff }}$ of the tunnel barriers The tunnel probability $\Gamma_{\imath}$ of barrier $\imath$ is renormalized to an effective tunnel probability $\Gamma_{\imath}^{\mathrm{eff}}$, which depends on the entire circuit

Nazarov's rules to compute the effective tunnel probabilities are as follows To each node and to each terminal of the circuit one assigns a vector $\boldsymbol{n}_{\imath}$ of unit length For a normal reservorr, $\boldsymbol{n}_{\imath}=(0,0,1)$ is at the north pole, for a superconducting reservoir, $\boldsymbol{n}_{\imath}=\left(\cos \phi_{\imath}, \sin \phi_{\imath}, 0\right)$ is at the equator For a node, $\boldsymbol{n}_{\imath}$ is somewhere on the northern hemisphere The vector $\boldsymbol{n}_{\imath}$ is called a "spectral vector", because it is a particular parameterization of the local energy spectrum If the tunnel barrier is located between spectral vectors $n_{1}$ and $n_{2}$, its effective tunnel probability is ${ }^{*}$

$$
\Gamma^{\mathrm{eff}}=\left(\begin{array}{ll}
n_{1} & n_{2}
\end{array}\right) \Gamma=\Gamma \cos \theta_{12},
$$

where $\theta_{12}$ is the angle between $n_{1}$ and $n_{2}$ The rule to compute the spectral vector of node $\imath$ follows from the continutty equation for the Green's function Let the index $k$ label the nodes or terminals connected to node $\imath$ by a single tunnel barrier (with tunnel probability $\Gamma_{k}$ ) Let the index $q$ label the nodes or terminals connected to $\imath$ by a diffusive conductor (with $L / l \equiv s_{q}$ ) The spectral vectors then satısfy the sum rule [65]

$$
\sum_{k}\left(n_{\imath} \times n_{k}\right) \Gamma_{k}+\sum_{q}\left(n_{\imath} \times n_{q}\right) \frac{\arccos \left(n_{\imath} n_{q}\right)}{s_{q} \sqrt{1-\left(n_{\imath} n_{q}\right)^{2}}}=0
$$

This is a sum rule for a set of vectors perpendicular to $\boldsymbol{n}_{\imath}$ of magnitude $\Gamma_{k} \sin \theta_{2 k}$ or $\theta_{\imath q} / s_{q}$, depending on whether the element connected to node $\imath$ is a tunnel

* It may happen that $\cos \theta_{12}<0$, in which case the effective tunnel probability 15 negative Nazarov has given an example of a four-terminal circutt with $\Gamma^{\text {eff }}<0$, so that the current through this banier flows in the duection opposite to the voltage drop [68] 


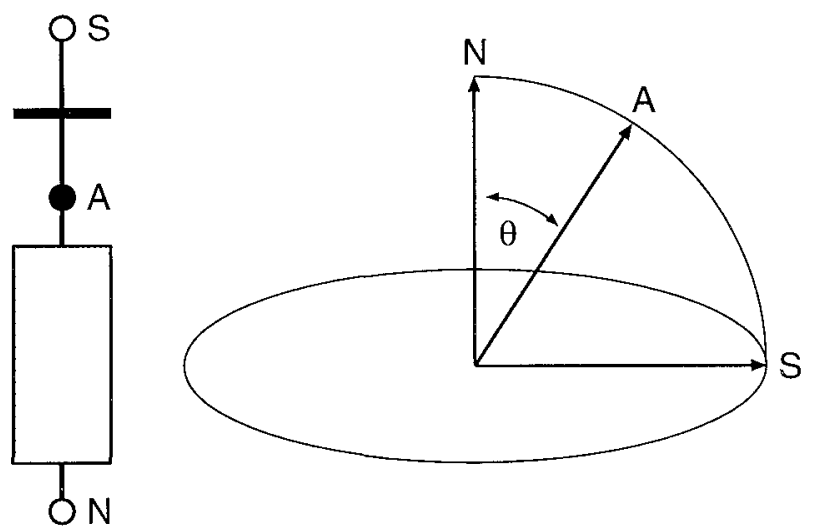

Fig. 13. At left: Circuit containing two terminals (open circles), one node (filled circle), and two elements: A diffusive conductor (shaded) and a tunnel barrier (black). At right: Spectral vectors associated with the terminals $\mathrm{N}, \mathrm{S}$ and with the node A.

barrier or a diffusive conductor. There is a sum rule for each node, and together the sum rules determine the spectral vectors of the nodes.

As a simple example, let us consider the system of section 5.2, consisting of one normal terminal $(\mathrm{N})$, one superconducting terminal $(\mathrm{S})$, one node (labeled $\mathrm{A}$ ), and two elements: A diffusive conductor (with $L / l \equiv s$ ) between $\mathrm{N}$ and $\mathrm{A}$, and a tunnel barrier (tunnel probability $\Gamma$ ) between $A$ and $S$ (see fig. 13). There are three spectral vectors, $n_{\mathrm{N}}, n_{\mathrm{S}}$, and $n_{\mathrm{A}}$. All spectral vectors lie in one plane. (This holds for any network with a single superconducting terminal.) The resistance of the circuit is given by $R=\left(h / 2 N e^{2}\right)\left(s+1 / \Gamma^{\mathrm{eff}}\right)$, with the effective tunnel probability

$$
\Gamma^{\mathrm{eff}}=\Gamma \cos \theta_{\mathrm{AS}}=\Gamma \sin \theta .
$$

Here $\theta \in[0, \pi / 2]$ is the polar angle of $\boldsymbol{n}_{\mathrm{A}}$. This angle is determined by the sum rule (5.36), which in this case takes the form

$$
\Gamma \cos \theta-\theta / s=0 .
$$

Comparison with section 5.2 shows that $\Gamma^{\mathrm{eff}}$ coincides with the effective tunnel probability $Q$ of eq. (5.21) in the limit $s \gg 1$, i.e. if one restricts oneself to the diffusive regime. That is the basic requirement for the application of the circuit theory.

Let us now consider the "fork junction" of fig. 14, with one normal terminal (N) and two superconducting terminals $\mathrm{S}_{1}$ and $\mathrm{S}_{2}$ (phases $\phi_{1} \equiv-\phi / 2$ and $\phi_{2} \equiv \phi / 2$ ). There is one node (A), which is connected to $\mathrm{N}$ by a diffusive conductor $(L / l \equiv$ $s)$, and to $S_{1}$ and $S_{2}$ by tunnel barriers $\left(\Gamma_{1}\right.$ and $\left.\Gamma_{2}\right)$. This structure was studied 


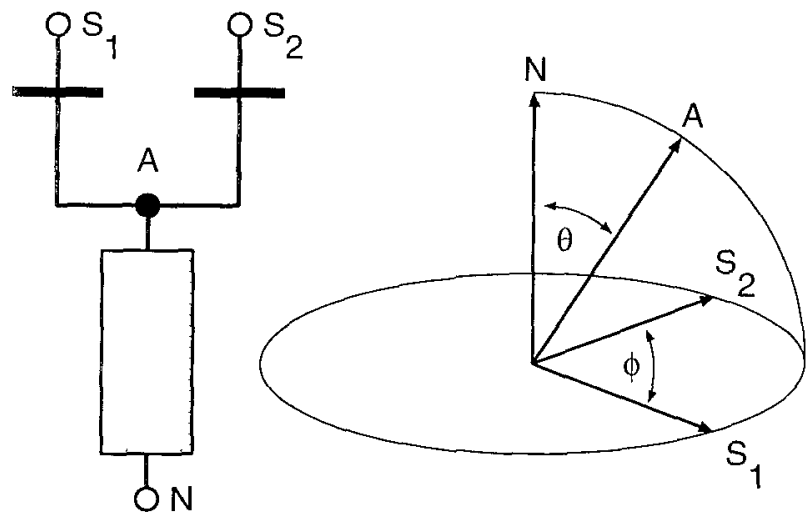

Fig 14. Circuit diagram and spectral vectors for a structure contaning one normal and two superconductung terminals (phase difference $\phi$ ).

theoretically by Hekking and Nazarov [53] and experimentally by Pothier et al. [69]. For simplicity, let us assume two identical tunnel barriers $\Gamma_{1}=\Gamma_{2} \equiv \Gamma$. Then the spectral vector $n_{\mathrm{A}}=(\sin \theta, 0, \cos \theta)$ of node A lies symmetrically between the spectral vectors of terminals $S_{1}$ and $S_{2}$. The sum rule (5.36) now takes the form

$$
2 \Gamma\left|\cos \frac{1}{2} \phi\right| \cos \theta-\theta / s=0 .
$$

Its solution determines the effective tunnel rate $\Gamma^{\mathrm{eff}}=\Gamma\left|\cos \frac{1}{2} \phi\right| \sin \theta$ of each of the two barriers in parallel, and hence the conductance of the fork junction,

$$
G=\frac{2 N e^{2}}{h}\left[s+\frac{1}{2}\left(\Gamma\left|\cos \frac{1}{2} \phi\right| \sin \theta\right)^{-1}\right]^{-1} .
$$

Two limiting cases of eqs. (5.39) and (5.40) are

$$
\begin{aligned}
& G=\left(2 N e^{2} / h\right)\left(s+\frac{1}{2} \Gamma^{-1}\left|\cos \frac{1}{2} \phi\right|^{-1}\right)^{-1}, \text { if } s \Gamma\left|\cos \frac{1}{2} \phi\right| \gg 1, \\
& G=\left(4 N e^{2} / h\right) s \Gamma^{2}(1+\cos \phi), \text { if } s \Gamma\left|\cos \frac{1}{2} \phi\right| \ll 1 .
\end{aligned}
$$

For $\phi=0$ (and $2 \Gamma \rightarrow \Gamma$ ) these expressions reduce to the results (5.23) and (5.24) for an NS junction with a single superconducting reservoir. The limit (5.42) agrees with the finite-temperature result of Hekking and Nazarov [53], if $s$ is replaced by $L_{c} / l$ and a series resistance is added due to the normal segment which is further than a correlation length from the NS interfaces. The possibility of a dependence of the conductance on the superconducting phase difference was noted also in other theoretical works, for different geometries [70-75].

The $\phi$-dependence of the conductance of a fork junction has recently been observed by Pothier et al. [69]. Some of their data is reproduced in fig. 15. The 


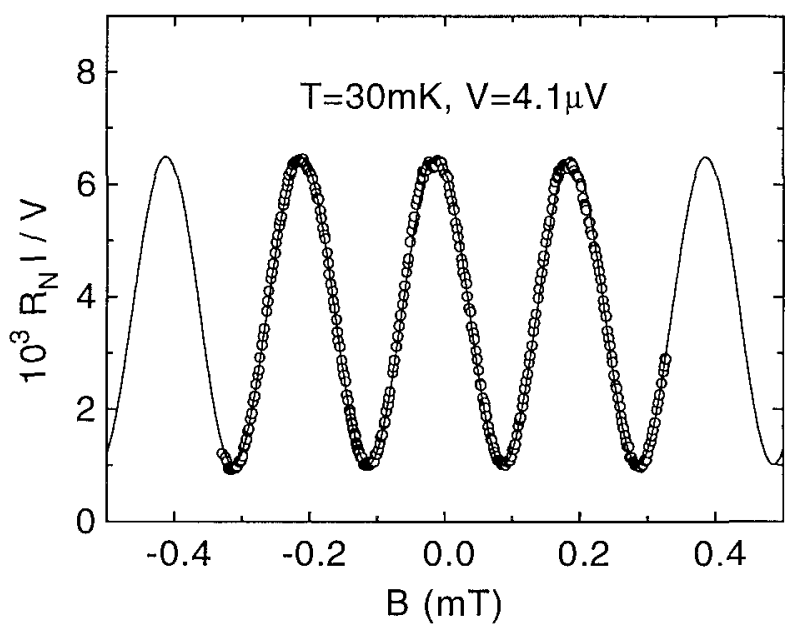

Fig 15 Conductance of a fork junction as a function of magnetic field, showing the dependence on the phase difference $\phi$ of the superconductor at two tunnel barriers The circles are measurements by Pothies et al [69] of the current $I$ through a $\mathrm{Cu}$ wire connected to an oxidized $\mathrm{Al}$ fork (normalstate resistance $R_{\mathrm{N}}=156 \mathrm{k} \Omega$ ) The applied voltage $V$ is sufficiently low that $I / V$ is close to the Inear-response conductance (The amplitude of the oscillations at $V=0$ is $394 \times 10^{-6} \Omega^{-1}$, somewhat larger than in the figure ) The solid curve is a cosine fit to the data The offset of maximum conductance from $B=0$ is attubuted to a small residual field in the cryostat (Courtesy of $\mathrm{H}$ Pothier )

conductance of a $\mathrm{Cu}$ wire attached to an oxidized $\mathrm{Al}$ fork oscillates as a function of the applied magnetic field. The period corresponds to a flux increment of $h / 2 e$ through the area enclosed by the fork and the wire, and thus to $\Delta \phi=2 \pi$. The experiment is in the regime where the junction resistance is dominated by the tunnel barriers, as in eq. (5.42).* The metal-oxide tunnel barriers in such structures have typically very small transmission probabilities $\left(\Gamma \simeq 10^{-5}\right.$ in ref. [69]), so that the regime of eq (5.41) is not easily accessible. Larger $\Gamma$ 's can be realized by the Schottky barrier at a semiconductor - superconductor interface It would be of interest to observe the crossover with increasing $\Gamma$ to the non-sinusoidal $\phi$-dependence predıcted by eq. (5.41), as a further test of the theory.

* Equation (5 42) provides only a qualitative description of the experiment, mainly because the motion in the arms of the foik is diffusive rathes than ballistic This is why the conductance minima in fig 15 do not go to zero A solution of the diffusion equation in the actual experimental geometry 1s required for a quantitative comparison with the theory [69] 


\section{Universal conductance fluctuations}

So far we have considered the average of the conductance over an ensemble of impurity potentials In fig 16 we show results of numerical simulations [39] for the varlance of the sample-to-sample fluctuations of the conductance, as a function of the average conductance in the normal state A range of parameters $L, W, l, N$ was used to collect this data, in the quasi-one-dimensional, metallic, diffusive regime $l<W<L<N l$ An ideal NS interface was assumed $(\Gamma=$ 1) The results for $\operatorname{Var} G_{\mathrm{N}}$ are as expected theoretically [34,35] for "universal conductance fluctuations" (UCF)

$$
\operatorname{Var} G_{N}=\frac{8}{15} \beta^{-1}\left(e^{2} / h\right)^{2}
$$

The index $\beta$ equals 1 in the presence and 2 in the absence of time-ieversal symmetry The $1 / \beta$ dependence of $\operatorname{Var} G_{\mathrm{N}}$ implies that the variance of the conductance fluctuations is reduced by a factor of two upon application of a magnetic field, as observed in the simulation (see the two dotted lines in the lower part of fig 16) The data for $\operatorname{Var} G_{\mathrm{NS}}$ at $B=0$ shows approximately a four-told increase over $\operatorname{Var} G_{\mathrm{N}}$ For $B \neq 0$, the simulation shows that $\operatorname{Var} G_{\mathrm{NS}}$ is essentially unaffected by a tıme-reversal-symmetry breakıng magnetıc field In contrast to the situation in the normal state, the theory for UCF in an NS junction is quite different for zero and for non-zero magnetic field, as we now discuss

In zero magnetic field, the conductance of the NS junction is given by eq (2 22), which is an expression of the form $A=\sum_{n} a\left(T_{n}\right)$ Such a quantity $A$ is called a linear statistic on the transmission eigenvalues The word "linear" refers to the fact that $A$ does not contain products of different $T_{n}$ 's The function $a(T)$ may well depend non-lınearly on $T$, as it does for $G_{\mathrm{NS}}$, where $a(T)$ is a rational function of $T$ The Landauer formula (2 23) for the normal-state conductance is also a linear statistic, with $a(T) \propto T$ It is a general theorem in random-matrix theory [76] that the varıance of a linear statıstic has a $1 / \beta$ dependence on the symmetry index $\beta$ Moreover, the magnitude of the variance is independent of the microscopic properties of the system (sample size, degree of disorder) This is Imry's fundamental explanation for UCF [28]

For a wire geometry, there exists a formula for the variance of an arbitrary linear statıst1c $[37,77,78]$,

$$
\begin{array}{r}
\operatorname{Var} A=-\frac{1}{2 \beta \pi^{2}} \int_{0}^{1} \mathrm{~d} T \int_{0}^{1} \mathrm{~d} T^{\prime}\left(\frac{\mathrm{d} a(T)}{\mathrm{d} T}\right)\left(\frac{\mathrm{d} a\left(T^{\prime}\right)}{\mathrm{d} T^{\prime}}\right) \\
\times \ln \left(\frac{1+\pi^{2}\left[x(T)+x\left(T^{\prime}\right)\right]^{-2}}{1+\pi^{2}\left[x(T)-x\left(T^{\prime}\right)\right]^{-2}}\right),
\end{array}
$$




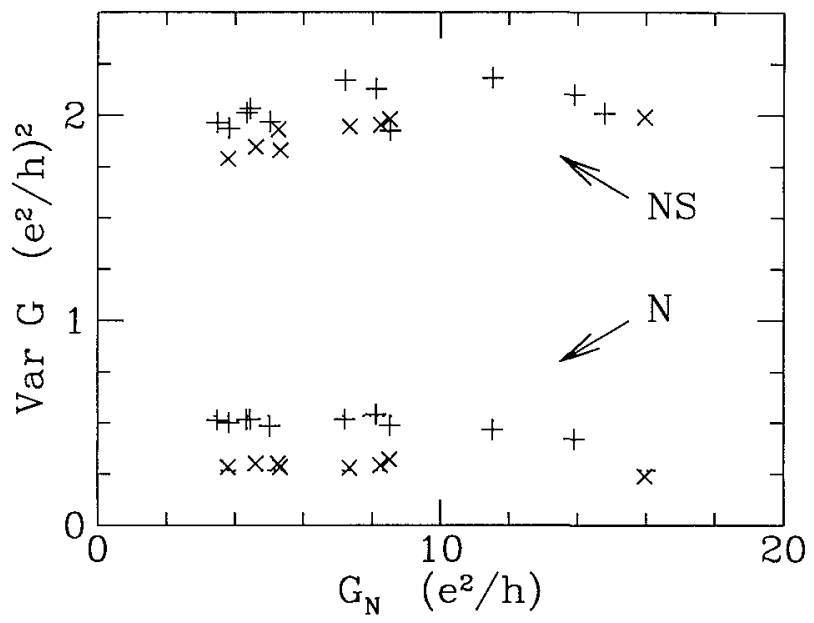

Fig 16 Numencal calculation of the variance of the fluctuations in $G_{\mathrm{N}}$ and $G_{\mathrm{NS}}$, as a function of the average $G_{\mathrm{N}}$ ( + for $B=0, \times$ for a flux of $10 \mathrm{~h} / \mathrm{e}$ ) Dotted lines are the analytical results fiom eqs (6 1) and (63) Note the absence of a factor of two reduction in $\operatorname{Var} G_{\mathrm{NS}}$ on applying a magnetıc field (From tef [39])

where $x(T)=\operatorname{arccosh} T^{-1 / 2} \quad$ In the normal state, substitution of $a(T)=$ $\left(2 e^{2} / h\right) T$ into eq $(62)$ reproduces the result $(61)$ In the NS junction, substitution of $a(T)=\left(4 e^{2} / h\right) T^{2}(2-T)^{-2}$ yields, for the case $\beta=1$ of zero magnetic field,

$$
\operatorname{Var} G_{\mathrm{NS}}=\frac{32}{15}\left(2-90 \pi^{-4}\right)\left(e^{2} / h\right)^{2}=430 \operatorname{Var} G_{\mathrm{N}}
$$

A factor of four between $\operatorname{Var} G_{\mathrm{NS}}$ and $\operatorname{Var} G_{\mathrm{N}}$ was estımated by Takane and Ebisawa [33], by an argument similar to that which we descilbed in section 4 for the weak-localization correction (A diagrammatic calculation by the same authors [79] gave a factor of six, presumably because only the dominant diagram was included) The numerical data in fig 16 is within $10 \%$ of the theoretical prediction (63) (upper dotted line) Similar numerical results for $\operatorname{Var} G_{N S}$ in zero magnetic field were obtaned in refs $[33,80]$

We conclude that UCF in zero magnetic field is basically the same phenomenon for $G_{\mathrm{N}}$ and $G_{\mathrm{NS}}$, because both quantities are linear statıstics for $\beta=1$ If time1eversal symmetry (TRS) is broken by a magnetic field, the situation is qualitatıvely diffetent For $G_{N}$, broken TRS does not affect the universalıty of the fluctuations, but merely reduces the variance by a factor of two No such simple behavio1 is to be expected for $G_{\mathrm{NS}}$, since it is no longer a linear statistic for $\beta=2$ That is a crucial distinction between eq (220) for $G_{\mathrm{NS}}$ and the Land- 
auer formula (223) for $G_{N}$, which remains a linear statistic regardless of whether TRS is broken or not This expectation [15] of an anomalous $\beta$ dependence of $\operatorname{Var} G_{\mathrm{NS}}$ was borne out by numerical simulations [39], which showed that the conductance fluctuations in an NS junction without TRS remain independent of disorder, and of approximately the same magnitude as in the presence of TRS (compare + and $x$ data points in the upper part of fig 16) An analytical theory remains to be developed

\section{Shot noise}

The conductance, which we studied in the previous sections, is the tume-averaged current $I$ divided by the applied voltage $V$ Time-dependent fluctuations $\delta I(t)$ in the current give additional information on the transport processes The zerofrequency norse power $P$ is defined by

$$
P=4 \int_{0}^{\infty} \mathrm{d} t\langle\delta I(t) \delta I(0)\rangle
$$

At zero temperature, the discreteness of the electron charge is the only source of fluctuations in time of the current These fluctuations are known as "shot norse", to distinguish them from the thermal nosse at non-zero temperature A furthe distinction between the two is that the shot-noise power is proportional to the applied voltage, whereas the thermal noise does not vanush at $V=0$ Shot noise is therefore an intrinsically non-equilibrium phenomenon If the transmission of an elementary charge $e$ can be regarded as a sequence of uncorrelated events, then $P=2 e|I| \equiv P_{\text {Poisson }}$ as in a Poisson process In this section we discuss, following ref [81], the enhancement of shot norse in an NS junction The enhancement originates from the fact that the current in the superconductor is carried by Cooper pairs in units of $2 e$ However, as we will see, a simple factor-of-two enhancement applies only in certain limiting cases

In the normal state, the shot-noise power (at zero temperature and infinitesimal applied voltage) is given by [82]

$$
P_{\mathrm{N}}=P_{0} \operatorname{Tr} t t^{\dagger}\left(1-t t^{\dagger}\right)=P_{0} \sum_{n=1}^{N} T_{n}\left(1-T_{n}\right),
$$

with $P_{0} \equiv 2 e|V|\left(2 e^{2} / h\right)$ Equation (72) is the mult1-channel generalization of earlier single-channel formulas [83,84] It is a consequence of the Paul principle that closed $\left(T_{n}=0\right)$ as well as open $\left(T_{n}=1\right)$ scattering channels do not fluctuate and therefore give no contribution to the shot noise In the case of a tunnel barrier, all transmission ergenvalues are small $\left(T_{n} \ll 1\right.$, for all $\left.n\right)$, so that the quadiatic terms in eq (72) can be neglected Then it follows from compatison with eq 
(223) that $P_{\mathrm{N}}=2 e|V| G_{\mathrm{N}}=2 e|I|=P_{\text {Poisson }}$ In contrast, for a quantum point contact $P_{\mathrm{N}} \ll P_{\text {Poisson }}$ Since on the plateaus of quantized conductance all the $T_{n}$ 's are either 0 or 1 , the shot noise is expected to be only observable at the steps between the plateaus [84] For a diffusive conductor of length $L$ much longer than the elastic mean free path $l$, the shot noise $P_{\mathrm{N}}=\frac{1}{3} P_{\text {Poisson }}$ Is one-third the Poisson norse, as a consequence of norseless open scattering channels [85,86]

The analogue of eq ( 72 ) for the shot-noise power of an NS junction is [81]

$$
P_{\mathrm{NS}}=4 P_{0} \operatorname{Tr} s_{\mathrm{he}} s_{\mathrm{he}}^{\dagger}\left(1-s_{\mathrm{he}} s_{\mathrm{he}}^{\dagger}\right)=P_{0} \sum_{n=1}^{N} \frac{16 T_{n}^{2}\left(1-T_{n}\right)}{\left(2-T_{n}\right)^{4}},
$$

where we have used eq ( 217 ) (with $\varepsilon=0$ ) to relate the scattering matrix $s_{\text {he }}$ for Andreev reflection to the transmission eigenvalues $T_{n}$ of the normal region This requires zero magnetic field As in the normal state, scattering channels which have $T_{n}=0$ or $T_{n}=1$ do not contribute to the shot norse However, the way in which partially transmitting channels contribute is entirely different from the nor mal state result ( 7 2)

Considei first an NS junction without disorder, but with an arbitrary transmission probability $\Gamma$ per mode of the interface In the normal state, eq (7 2) yields $P_{\mathrm{N}}=(1-\Gamma) P_{\text {Poisson }}$, implying full Poisson noise for a high tunnel barrier $(\Gamma \ll 1)$ For the NS junction we find from eq $(73)$

$$
P_{\mathrm{NS}}=P_{0} N \frac{16 \Gamma^{2}(1-\Gamma)}{(2-\Gamma)^{4}}=\frac{8(1-\Gamma)}{(2-\Gamma)^{2}} P_{\text {Poisson }}
$$

where in the second equality we have used eq (222) This agrees with results obtained by Khlus [83], and by Muzykantskı̆ and Khmel'nitskı̆ [87], using different methods If $\Gamma<2(\sqrt{2}-1) \approx 083$, one observes a shot noise above the Poisson noise For $\Gamma \ll 1$ one has

$$
P_{\mathrm{NS}}=4 e|I|=2 P_{\text {Polsson }} \text {, }
$$

which is a doubling of the shot-noise power divided by the current with respect to the normal-state result This can be inteipreted as uncorrelated current pulses of $2 e$-charged particles

Consider next an NS junction with a disordered normal region, but with an ideal interface $(\Gamma=1)$ We may then apply the formula ( 310$)$ for the average of a linear statıstic on the transmission eigenvalues to eqs (2 22) and (7 3) The result is

$$
\frac{\left\langle P_{\mathrm{NS}}\right\rangle}{\left\langle G_{\mathrm{NS}}\right\rangle}=\frac{2}{3} \frac{P_{0}}{2 e^{2} / h} \Rightarrow\left\langle P_{\mathrm{NS}}\right\rangle=\frac{4}{3} e|I|=\frac{2}{3} P_{\text {Poisson }}
$$

Equation (7 6) is twice the result in the normal state, but still smaller than the Poisson noise Corrections to (7 6) are of lower order in $N$ and due to quantuminterference effects [88] 


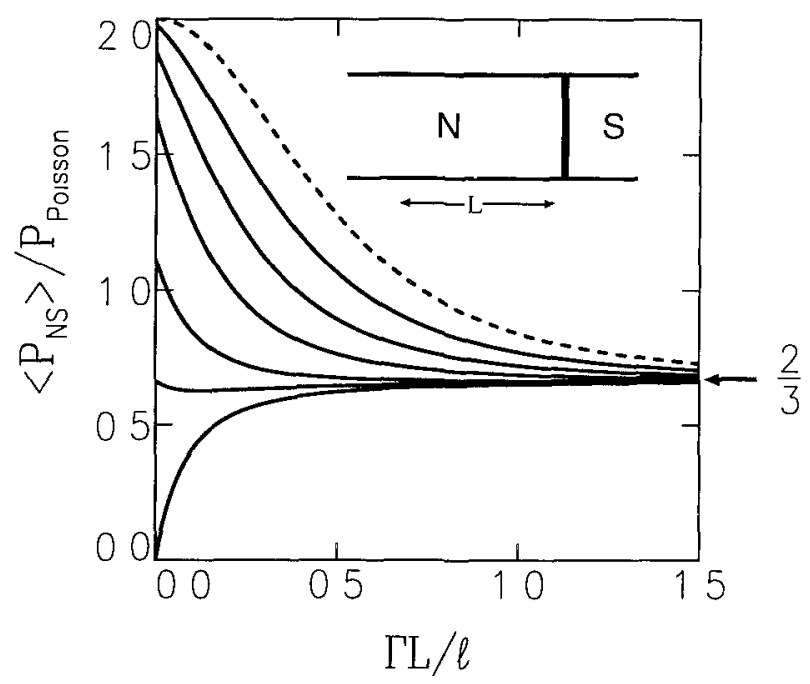

Fig 17 The shot noise power of an NS junction (1n units of $P_{\text {Poisson }} \equiv 2 e|I|$ ) as a function of the length $L$ (in unts of $l / \Gamma$ ) for barrier transparencies $\Gamma=10908060402$ from bottom to top The dashed curve gives the limiting result for $\Gamma \ll 1$ For $L=0$ the norse power varies as a function of $\Gamma$ according to eq (7 4) between doubled shot noise $\left(\left\langle P_{\mathrm{NS}}\right\rangle=4 e|I|\right)$ for a high banier $(\Gamma \ll 1)$ and zero in the absence of a barrier $(\Gamma=1)$ For $L \rightarrow \infty$ the norse power approaches the limiting value $\left\langle P_{\mathrm{NS}}\right\rangle=\frac{4}{3} e|I|$ fot each $\Gamma$ (From ref [81])

Finally, consider an NS junction which contains a disordered normal region (length $L$, mean free path $l$ ) as well as a non ideal interface The scaling theory of section 52 has been applied to this problem in ref [81] Results are shown in fig 17 , where $\left\langle P_{\mathrm{NS}}\right\rangle / P_{\text {Porsson }} 1$ plotted against $\Gamma L / l$ for varıous $\Gamma$ Note the crossover from the ballistic result (7 4) to the diffusive result (7 6) For a high barrier $(\Gamma \ll 1)$, the shot noise decreases from twice the Poisson norse to twothirds the Poisson noise as the amount of disorder increases

\section{Conclusion}

We have reviewed a scattering approach to phase coherent transport accross the interface between a normal metal and a superconductor For the reflectionless tunneling phenomenon, the complete equivalence has been demonstrated to the non-equilibrium Green's function approach (The other effects we discussed have so far mainly been treated in the scattering approach ) Although mathematically 
equivalent, the physical picture offered by the two approaches is quite different We chose to focus on the scattering approach because it makes direct contact with the quantum interference effects studied extensively in the normal state The same technıques used for weak localızation and universal conductance fluctuations in normal conductors could be used to study the modifications by Andieev reflection in an NS junction

In the limit of zero voltage, zero temperature, and zero magnetic field, the transport properties of the NS junction are determined entirely by the transmission eigenvalues $T_{n}$ of the normal region A scaling theory for the distribution of the $T_{n}$ 's then allows one to obtain analytical results for the mean and varı ance of any observable of the form $A=\sum_{n} a\left(T_{n}\right)$ The conductance is of this form, as well as the shot-noise power The only difference with the normal state is the functional form of $a(T)$ (polynomial in the normal state, rational function for an NS junction), so that the general results of the scalıng theory [valid for any function $a(T)]$ can be applied at once At finite $V, T$, or $B$, one needs the entire scattering matrix of the normal region, not just the transmission eigenvalues This poses no difficulty for a numerical calculation, as we have shown in several examples However, analytical progress using the scattering approach becomes cumbersome, and a diagrammatic Green's function calculation is more efficient

\section{Acknowledgments}

It is a pleasure to acknowledge my collaborators in this research R A Jalabert, M J M de Jong, I K Marmorkos, J A Melsen, and B Rejaeı Financial support was provided by the "Nederlandse organisatie voor Wetenschappelıjk Onderzoek" (NWO), by the "Strchtıng voor Fundamenteel Onderzoek der Materie" (FOM), and by the European Community I have greatly benefitted from the insights of Yu V Nazarov Permission to reproduce the experimental figs 5, 7, and 15 was kındly given by $\mathrm{K}-\mathrm{M} \mathrm{H}$ Lenssen, A W Kleınsasser, and H Pothıe, respectively

\section{Note added February 1995}

The theory of section 4 has been extended to non-zero voltage and magnetic field by PW Brouwer and the author (Phys Rev B 52 (1995) R3868) The results are $\delta G_{\mathrm{NS}}(V=0, B \neq 0)=\frac{1}{3} e^{2} / h, \delta G_{\mathrm{NS}}(V \neq 0, B=0)=\frac{2}{3} e^{2} / h, \delta G_{\mathrm{NS}}(V \neq$ $0, B \neq 0)=0$ The disagreement with the numerical simulations discussed in sectron 4 is due to an insufficiently large system size 


\section{References}

[1] A F Andreev, Zh Eksp Teor Fiz 46 (1964) 1823 [Sov Phys JETP 19 (1964) 1228]

[2] C W J Beenakker and H van Houten, Solid State Phys 44 (1991) 1

[3] C J Lambert, J Phys Condens Matter 5 (1993) 707

[4] C J Lambert, VC Hul, and S J Robinson, J Phys Condens Matter 5 (1993) 4187

[5] VT Petrashov, VN Antonov, P Delsing, and T Claeson, Phys Rev Lett 70 (1993) 347

[6] T M Klapwijk, Physica B 197 (1994) 481

[7] C W J Beenakker, Phys Rev Lett 67 (1991) 3836, 68 (1992) 1442(E)

[8] C W J Beenakker, in Transport Phenomena in Mesoscopı Systems, H Fukuyama and T Ando, eds (Spunger, Berlın, 1992)

[9] The contribution of scattering inside the superconductor to the resistance of an NS function has been studied extensively, see for example A B Pippard, J G Shepherd, and D A Tindall Pioc R Soc London A 324 (1971) 17, A Schmid and G Schon, J Low Temp Phys 20 (1975) 207 T Y Hsiang and J Clarke, Phys Rev B 21 (1980) 945

[10] PG de Gennes, Superconductivity of Metals and Alloys (Benjamin, New York, 1966)

[11] K K Likharev, Rev Mod Phys 51 (1979) I01

[12] GE Blonder, M Tinkham, and T M Klapw1jk, Phys Rev B 25 (1982) 4515

[13] C J Lambert, J Phys Condens Matter 3 (1991) 6579

[14] Y Takane and H Ebisawa, J Phys Soc Jpn 61 (1992) 1685

[15] C W J Beenakker, Phys Rev B 46 (1992) 12841

[16] A L Shelankov, F1z Tverd Tela 26 (1984) 1615 [Sov Phys Solıd State 26 (1984) 981]

[17] A V Zaŭtsev, Zh Eksp Teor Fiz 86 (1984) 1742 [Sov Phys JETP 59 (1984) 1742]

[18] H van Houten and C W J Beenakker, Physica B 175 (1991) 187

[19] A V Zaütsev, Zh Eksp Teor Fiz 78 (1980) 221, 79 (1980) 2016(E) [Sov Phys JETP 51 (1980) $111,52(1980) 1018(\mathrm{E})]$

[20] M Buttıker, Phys Rev B 41 (1990) 7906

[21] M Buttıker, IBM J Res Dev 32 (1988) 63

[22] L I Glazman and K A Matveev, Pıs'ma Zh Eksp Teor Fiz 49 (1989) 570 [JETP Lett 49 (1989) 659]

[23] CWJ Beenakker and $\mathrm{H}$ van Houten, in Single Electron Tunneling and Mesoscopic Devices, $\mathrm{H}$ Koch and H Lubbig, eds (Springer, Beılın, 1992)

[24] V A Khlus, A V Dyomın, and A L Zazunov, Physica C 214 (1993) 413

[25] I A Devyatov and M Yu Kupriyanov, P1s'ma Zh Eksp Teor Fiz 52 (1990) 929 [JETP Lett $52(1990) 311]$

[26] FW J Hekkıng, L I Glazman, K A Matveev, and R I Shekhter, Phys Rev Lett 70 (1993) 4138

[27] O N Dorokhov, Solid State Comm 51 (1984) 381

[28] Y Imry, Europhys Lett 1 (1986) 249

[29] J B Pendry, A MacKınnon, and PJ Roberts, Proc R Soc London A 437 (1992) 67

[30] Yu V Nazarov, Phys Rev Lett 73 (1994) 134

[31] A F Andieev, Zh Eksp Teor Fiz 51 (1966) 1510 [Sov Phys JETP 24 (1967) 1019]

[32] S N Artemenko, A F Volkov, and A V Zaŭtsev, Solid State Comm 30 (1979) 77 I

[33] Y Takane and H Ebisawa, J Phys Soc Jpn 61 (1992) 2858

[34] A D Stone, PA Mello, K A Muttahib, and J -L Pichard, in Mesoscoptc Phenomena in Solid, B L Al'tshuler, PA Lee, and R A Webb, eds (North Holland, Amsterdam, 199I)

[35] PA Mello and A D Stone, Phys Rev B 44 (1991) 3559

[36] C W J Beenakke1, Phys Rev B 49 (1994) 2205

[37] A M S Macêdo and J T Chalker, Phys Rev B 49 (1994) 4695 
[38] Y Takane and H Otan [J Phys Soc Jpn 63 (1994) 3361] find $\delta G_{\mathrm{NS}}=\frac{4}{3} e^{2} / h$, in slight disagreement with eq (4 6)

[39] I K Marmorkos, C W J Beenakker, and R A Jalabert, Phys Rev B 48 (1993) 2811

[40] K -M H Lenssen, PC A Jeekel, C J PM Harmans, J E Mooy, M R Leys, J H Wolter, and M C Holland, in Coulomb and Interference Effects in Small Electronic Structures, D C Glattl and $M$ Sanquer, eds (Editions Fiontieles, Gif sur-Yvette, 1994)

[41] A Kastalsky, A W Klemsasser, L H Gieene, R Bhat, FP Millken, and J P Harbıson, Phys Rev Lett 67 (1991) 3026

[42] C Nguyen, H Kroemer, and E L Hu, Phys Rev Lett 69 (1992) 2847

[43] R G Manı, L Ghenum, and T N Thers, Phys Rev B 45 (1992) 12098

[44] N Agrait, J G Rodrigo, and S Viewa, Phys Rev B 46 (1992) 5814

[45] P Xiong, G Xiao, and R B Labowitz, Phys Rev Lett 71 (1993) 1907

[46] K -M H Lenssen, L A Westelling, PC A Jeekel, C J P M Harmans, J E Mool], M R Leys, W van der Vleuten, J H Wolter, and S P Beaumont, Physica B 194-196 (1994) 2413

[47] S J M Bakker, E van der Druft, TM Klapwık, H M Jaegeı, and S Radelaar, Phys Rev B 49 (1994) 13275

[48] PH C Magnee, N van de Post, PH M Kooıstra, B J van Wees, and TM Klapwıjk, Phys Rev B 50 (1994) 4594

[49] Y Takane and H Ebisawa J Phys Soc Jpn 62 (1993) 1844

[50] B J van Wees, P de Vries, P Magnee, and T M Klapwi1k, Phys Rev Lett 69 (1992) 510

[51] Y Takane and H Ebısawa, J Phys Soc Jpn 61 (1992) 3466

[52] A F Volkov, A V Zaĭtsev, and T M Klapwijk, Physica C 210 (1993) 21

[53] FW J Hekkıng and Yu V Nazarov, Phys Rev Lett 71 (1993) 1625, Phys Rev B 49 (1994) 6847

[54] C W J Beenakker, B Rejae1, and J A Melsen, Phys Rev Lett 72 (1994) 2470

[55] A V Zaŭtsev, Pis'ma Zh Eksp Teor Fiz 51 (1990) 35 [JETP Lett 51 (1990) 41], Physica C 185-189 (1991) 2539

[56] A F Voikov and TM Klapwijk, Phys Lett A 168 (1992) 217

[57] A F Volkov, Pis'ma Zh Eksp Teor Fiz 55 (1992) 713 [JETP Lett 55 (1992) 746], Phys Lett A 174 (1993) 144

[58] A L Shelankov, P1s'ma Zh Eksp Teor Fiz 32 (1980) 122 [JETP Lett 32 (1980) 111]

[59] PA Mello and J -L Pichard, Phys Rev B 40 (1989) 5276

[60] O N Dorokhov, Pis'ma Zh Eksp Teor Fiz 36 (1982) 259 [JETP Lett 36 (1982) 318]

[61] P A Mello, P Pereyra, and N Kumar, Ann Phys 181 (1988) 290

[62] J A Melsen and C W J Beenakkeı, Physıca B 203 (1994) 219

[63] D R Heslinga, S E Shafranjuk, H van Kempen, and TM Klapwijk, Phys Rev B 49 (1994) 10484

[64] A F Volkov, Phys Lett A 187 (1994) 404

[65] Yu V Nazarov Phys Rev Lett 73 (1994) 1420

[66] A I Larkın and Yu N Ovchınnkov, Zh Eksp Teor Fiz 68 (1975) 1915, 73 (1977) 299 [Sov Phys JETP 41 (1975) 960, 46 (1977) 155]

[67] M Yu Kupriyanov and VF Lukıchev, Zh Eksp Teor Fiz 94 (1988) 139 [Sov Phys JETP 67 (1988) 1163]

[68] Yu V Nazaıov, contribution at the NATO Adv Res Workshop on "Mesoscopic Superconduct 1vity" (Karlsruhe, May 1994)

[69] H Pothrer, S Guéron, D Esteve, and M H Devoret, Phys Rev Lett 73 (1994) 2488

[70] B Z Spıvak and D E Khmel'nutskıu,, Pıs'ma Zh Eksp Teor Fiz 35 (1982) 334 [JETP Lett 35 (1982) 412]

[71] B L Al'tshuleı and BZ Spivak, Zh Eksp Teor Fiz 92 (1987) 609 [Sov Phys JETP 65 
(1987) 343]

[72] H Nakano and H Takayanagı, Solıd State Comm 80 (1991) 997

[73] S Takagi, Solıd State Comm 81 (1992) 579

[74] C J Lambert, J Phys Condens Matter 5 (1993) 707

[75] V C Hui and C J Lambert, Europhys Lett 23 (1993) 203

[76] C W J Beenakker, Phys Rev Lett 70 (1993) 1155, Phys Rev B 47 (1993) 15763

[77] C W J Beenakker and B Rejaeı, Phys Rev Lett 71 (1993) 3689, Phys Rev B 49 (1994) 7499

[78] J T Chaiker and A M S Macêdo, Phys Rev Lett 71 (1993) 3693

[79] Y Takane and H Ebisawa, J Phys Soc Jpn 60 (1991) 3130

[80] J Bruun, VC Hu1, and C J Lambert, Phys Rev B 49 (1994) 4010

[81] M J M de Jong and C W J Beenakker, Phys Rev B 49 (1994) 16070

[82] M Buttker, Phys Rev Lett 65 (1990) 2901, Phys Rev B 46 (1992) 12485

[83] VA Khlus, Zh Eksp Teor FIZ 93 (1987) 2179 [Sov Phys JETP 66 (1987) 1243]

[84] G B Lesovik, Pis'ma Zh Eksp Teor Fiz 49 (1989) 513 [JETP Lett 49 (1989) 592]

[85] C W J Beenakker and M Buttker, Phys Rev B 46 (1992) 1889

[86] K E Nagaev, Phys Lett A 169 (1992) 103

[87] B A Muzykantskiı and D E Khmel'nitskı̌̆, Physıca B 203 (1994) 238

[88] M J M de Jong and C W J Beenakket, Phys Rev B 46 (1992) 13400 